\title{
The Development of Adaptive Optics and Its Application in Ophthalmology
}

\author{
Gopal Swamy Jayabalan and Josef F. Bille
}

\subsection{Introduction}

Wavefront technology was originally developed nearly 50 years ago for astronomical applications. It was used to measure wavefront distortions that occurred when light traveling through the atmosphere entered an optical telescope. By applying adaptive optical closed-loop controls the speckle patterns of the star images could be improved towards diffraction-limited performance. Most of the technology was developed in association with research towards anti-missile defense systems in the late nineteen-hundred seventies. The same technology can be used to correct the ocular imperfections.

Historically, refractive errors of the human eye were corrected by glasses, contact lenses and recently with excimer laser surgery. However, such corrections were limited to the compensation of only the lower order aberrations such as myopia, hyperopia, or regular astigmatism. Indeed the optical system of the human eye as a genuine optical system generates more complex

G. S. Jayabalan

Heidelberg Engineering GmbH,

Heidelberg, Germany

University of Heidelberg, Heidelberg, Germany

J. F. Bille $(\bowtie)$

University of Heidelberg, Heidelberg, Germany distortions to the retinal images, the so-called higher-order aberrations. These aberrations are unique for the very particular eye of the patient. Under daylight vision conditions the pupil of the human eye is small, e.g. $2-3 \mathrm{~mm}$ diameter, so that the light travels essentially along the optical axis of the human eye (Fig. 16.1, left image). Under these conditions higher-order aberrations are limited so that a sharp retinal image is formed. Under twilight vision conditions, the pupil of the human eye dilates to approx. 5-7 mm diameter resulting in increased importance of higher-order aberrations (Fig. 16.1, right image). These higher-order aberrations result in considerable distortions to the retinal image as a considerable part of the light is transmitted through marginal areas of the human eye, away from the optical axis. These image distortions impair the visual acuity even in patients with normal vision (20/20 visual acuity) considerably.

These complex distortions can be assessed with the wavefront technology. In recent years different wavefront sensors based on several principles have been developed, and the most important ones being Tscherning ray tracing and Shack-Hartmann sensors. More recently, the application of wavefront sensing for preoperative evaluation of refractive surgical procedures has been proposed. Adaptive optical closed loop systems can be used to subjectively measure and compensate the higher-order optical 


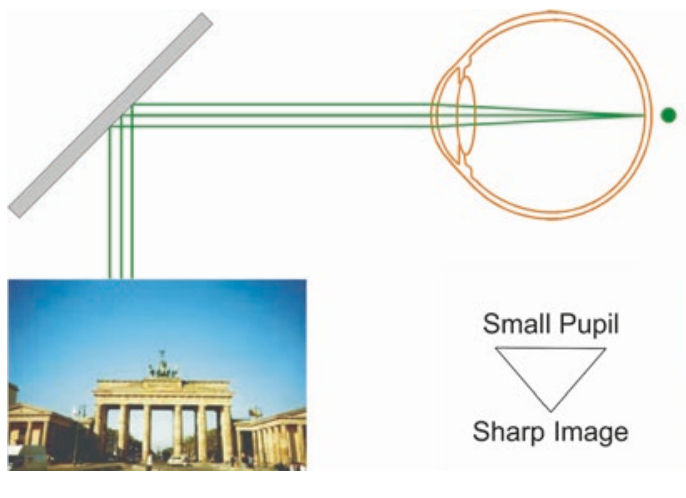

Fig. 16.1 Left: Daylight vision, Right: Twilight vision

aberrations of the human eye to guide the surgeon in the selection of parameters of the procedure.

\subsection{Brief History of Adaptive Optics}

Starting in 1978, the principle of wavefront measurement and compensation of wavefront aberrations was adopted at the University of Heidelberg for ophthalmic applications. The technique is based on Shack-Hartmann sensing, measuring the optical path of light rays through the eye to detect all aberrations at all points in the optical system of the human eye. Adaptive optical systems were developed to measure and compensate wave aberrations of the human eye with closedloop control [1-2].

As early as 1982 at the sixth International Conference on Pattern Recognition (ICPR) in Munich, Germany, wavefront sensing and adaptive optical closed-loop control was proposed for aberration-free imaging and vision testing: "The system essentially provides an elimination of optical eye aberrations which diminish the fundus image quality (Table 16.1). On the other hand by active focus control and/or wavefront sensing the aberrations of the human eye like astigmatism of the cornea and spherical aberration of the lens can be measured" [1]. In another publication, the concept of achieving 20/10 visual acuity by adaptive optical visual stimulus generation was described: "In the apparatus of this invention the illuminating laser beam is generally

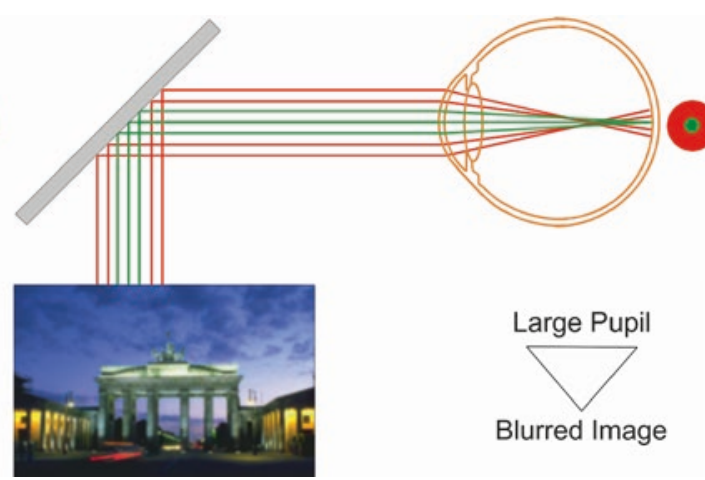

Table 16.1 History of adaptive optics closed-loop control

\begin{tabular}{l|l}
\hline 1976 & Adaptive optics in astronomy \\
\hline 1982 & $\begin{array}{l}\text { Bille et al.: Image restoration by adaptive } \\
\text { optical phase compensation. sixth ICPR }\end{array}$ \\
\hline 1986 & $\begin{array}{l}\text { Bille: U.S Patent, 4,579,430_-Method and } \\
\text { apparatus for forming an image of the ocular } \\
\text { fundus }\end{array}$ \\
\hline 1986 & $\begin{array}{l}\text { Clafin et al.: Configuring an electrostatic } \\
\text { membrane mirror by least squares fitting with } \\
\text { analytically derived influence functions [3] }\end{array}$ \\
\hline
\end{tabular}

widened to a diameter of between $3 \mathrm{~mm}$ and 4 $\mathrm{mm}$, in exceptional cases even still wider, and by compensating for all existing aberrations it is possible to focus the laser beam on a spot of a minimal diameter between 2 and 3 micrometers on the retina. This permits a resolution of more than 5000 image points per scan line, that is, it is possible for example to resolve and represent individual receptors in the fovea. Since the use of optical image focusing under adaptive control produces data on the wavefront of the imaging laser beam, the apparatus of this invention enables the refractive index profile within the eye to be reconstructed, permitting for the first time an automatic determination of the refraction at high accuracy" [2].

At the same time, an adaptive optical control system was devised and built based on modal actuator control (Fig. 16.2). In modal phase compensation, the wavefront aberration is expanded into an orthonormal system based on Zernike polynomials. In addition, the original concept included a Karhunen-Loève wave expansion, in 


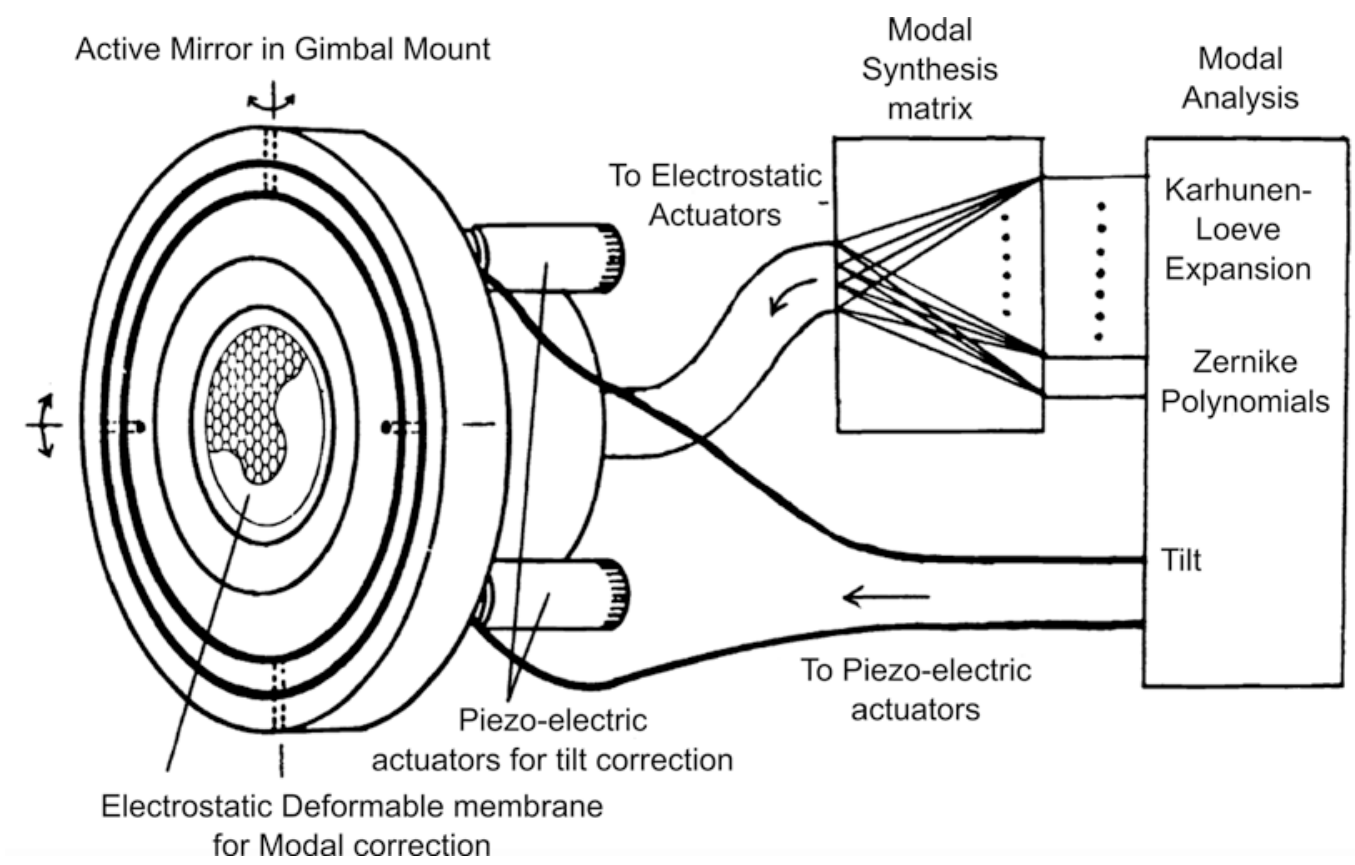

Fig. 16.2 Closed loop adaptive optical system with modal actuator control (adapted by permission from SPIE: [1, 2])

order to account for partial wavefront distortions with high spatial frequency content [4].

In 1989, Dreher along with Bille and Weinreb attempted to measure and correct monochromatic aberrations using an active mirror to correct ocular aberrations and provided improved depth resolution retinal images using scanning laser ophthalmoscope (SLO) [5]. The clinical adoption of Shack-Hartmann wavefront sensor to measure eyes wave aberration was demonstrated in early 90s at the University of Heidelberg, in Bille's laboratory with Liang working as a graduate student [6]. This led to the key development of closed-loop adaptive optics systems for ophthalmology. Later, Liang with Williams at the University of Rochester built the first closed-loop adaptive optics system that could correct higherorder aberrations of the eye and achieve a supernormal vision and visualization of single cells in the human retina [7]. Thereafter, wavefrontguided laser refractive surgery was introduced as a clinical treatment for refractive correction [8]. Although there are many methods to measure the ocular aberrations, Shack-Hartmann is considered the finest method to precisely measure the aberra- tions in the human eye and is generally employed in clinical aberrometers. The wavefront technology advancement in recent years has advanced to produce accurate measurements and diagnoses of higher-order aberrations. And this leads to wavefront designed glasses, contact lenses, intraocular implants, and wavefront-guided laser vision correction. Also, fundus cameras, SLO, Optical coherence tomography (OCT) and two-photon ophthalmoscopy (TPO) have incorporated adaptive optics to achieve diffraction-limited imaging system [9-11].

\subsection{Higher-Order Aberrations}

Any imperfection in the eye will lead to distorted images and decrease in visual performance. This is commonly referred to as optical or wavefront aberrations. The lower order aberrations and higher-order aberrations are the two types of wavefront aberrations of the eye. The lower order aberrations such as myopia, hyperopia, and astigmatism can be corrected with glasses, contact lenses or refractive surgery. Higher-order 
aberrations are the imperfections that cannot be corrected by these technologies. There are some degrees of higher-order aberrations present in each eye and this can be measured using the wavefront technology. The wavefront aberrations of the human eye are generally defined mathematically by a series of Zernike polynomials (for a detail description of Zernike polynomials, see Chap. 18).

In Zernike polynomials, the lower order aberrations are represented as the second-order and the higher-order aberrations are above secondorder. For example, the coma and trefoil are the third-order aberrations and the spherical aberrations are fourth-order Zernike terms. The lower order aberrations contribute $85 \%$ of all aberrations in the eye, whereas the higher-order aberrations contribute only $15 \%$ of the aberrations. The higher-order aberrations are more complex than the lower order aberrations and these aberrations results in difficulty seeing at night, glare, halos, blurring, starburst patterns or double vision [12]. These aberrations can be measured using the wavefront sensor. The refractive index and linear path variations are measured with a wavefront sensor and these generate a map that shows relevant retardation that a plane wave undergoes as it traverses the optics of the eye. The clinicians are familiar with the wavefront map and the Zernike polynomial expansion. The coefficient of each Zernike term reveals the total root mean square (RMS) error. In Fig. 16.3, the pseudo-3D graphics of the Zernike functions is shown.

\subsection{Principle of Aberration Measurement}

In recent years, basically, three types of aberration measurement devices have been developed: The thin beam ray tracing aberrometer, the Tscherning aberrometer, and the Shack-Hartmann method. In Fig. 16.4, the principle of operation of the Shack-Hartmann wavefront sensor is demonstrated. On the left-hand side, the processing of an ideal plane wave is depicted. The incident plane wave results in a square grid of spots in the focal plane of the micro-lens array. On the right-hand side, the imaging of a distorted wave is shown. The distorted wavefront causes lateral displacements of the spots on the CCD array. From the spot pattern, the shape of the incident wavefront can be reconstructed based on appropriate curve fitting algorithms. More than 25 years ago the first detailed study of the application of wavefront technology for the assessment of the refractive properties of the human eye was performed. From the wavefront measurements, Zernike coefficients were calculated and the wavefronts emerging from the eyes tested were reconstructed. Figure 16.5 shows the equal level contour maps of a human eye $[6,13,14]$. On the left-hand side of Fig. 16.5 the overall wavefront is presented, whereas on the right-hand side only the higher-order aberrations, i.e. the third- and fourth-order Zernike coefficients are depicted. In this work, the phase error that cannot be corrected by conventional spectacles was specified as the higher-order aberrations of the eye. In Fig. 16.6 the principle of the measuring process of the WaveScan ${ }^{\mathrm{TM}}$ instrument is shown. The ideal wavefront is represented as a regular grid of spots coded with green color. The distorted wavefront is given by an irregular grid of spots coded with red color.

\subsection{Definitions of Optical Imaging Quality}

For the description of the performance of an optical system, there are several parameters in use. Some of them are applied to the human eye as well. A short overview of some scales used in ophthalmology will be given in this section.

\subsubsection{Root Mean Square (RMS)}

The RMS of the wavefront is a very simple criterion. It is nothing but the integrated root mean square of the differences between the wavefront surface and the mean value of the surface. The complex phenomenon of aberration is packed 


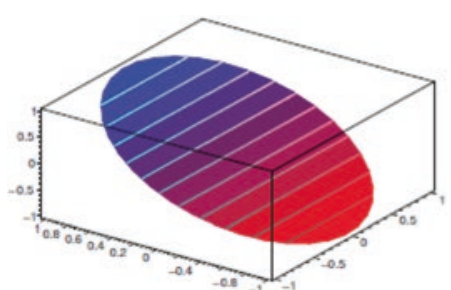

\section{$Z_{1}$ Tilt in x-direction}

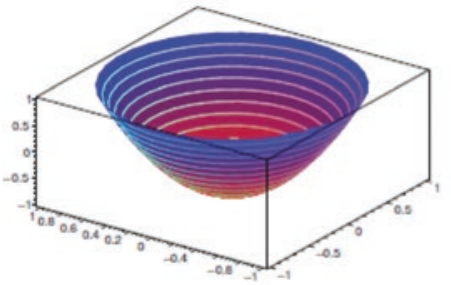

$Z_{4}$ Defocus

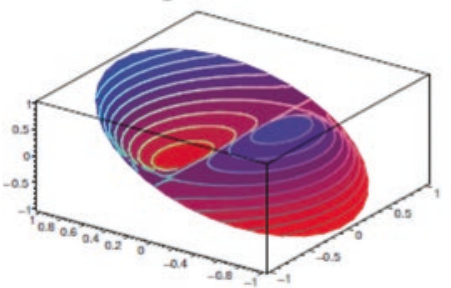

$Z_{7}$ Coma in $\mathrm{x}$
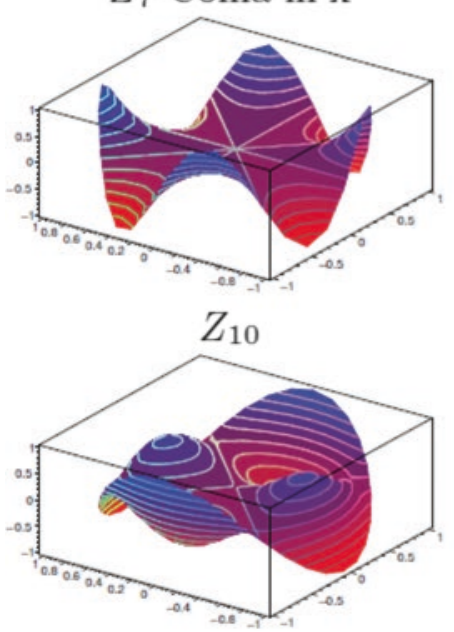

$Z_{13}$

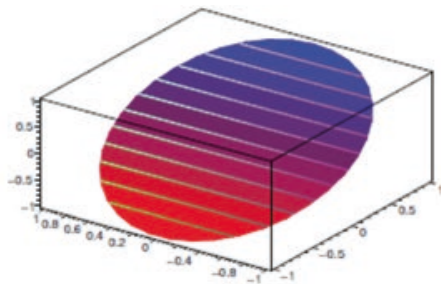

$Z_{2}$ Tilt in y-direction
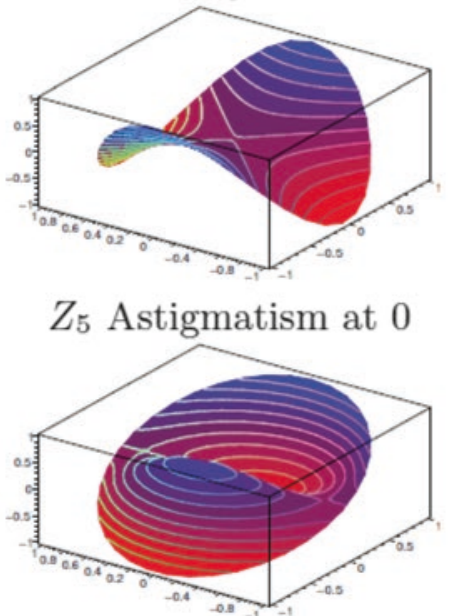

$Z_{8}$ Coma in $\mathrm{y}$
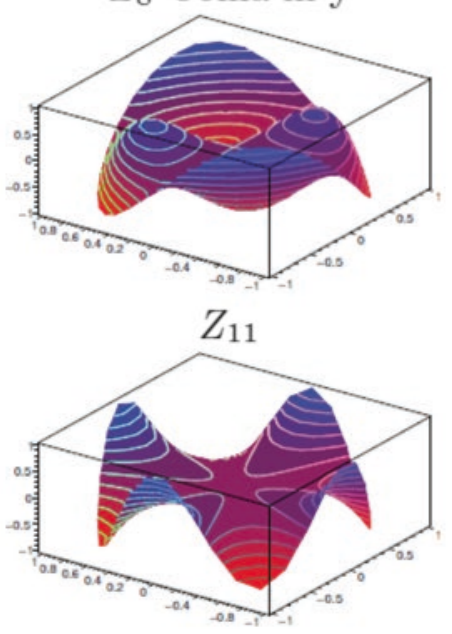

$Z_{14}$

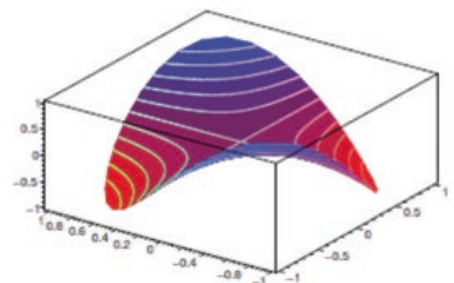

$Z_{3}$ Astigmatism at 45
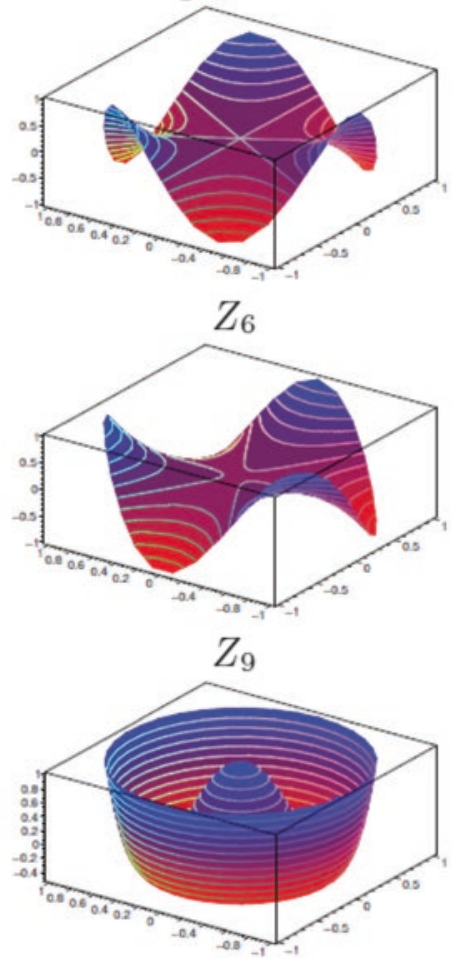

$Z_{12}$ Spherical aberration

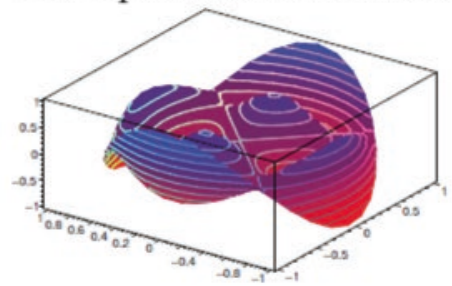

$Z_{25}$ Example of 6 th order

Fig. 16.3 Zernike polynomials up to fourth order and an example of sixth order control

into a single number. This makes it so convenient for ophthalmology. The RMS can be calculated directly from the Zernike polynomials.

For the calculation of RMS, we refer to Zernike polynomials of second order minimum. The zero order is not measured at all. The first order gives information only about the tilt, which is connected to the position of the eye. It does not supply any information about the characteristics of the eye itself. The Zernike polynomials are orthogonal and the zero order term is set to zero. So the mean value of the wavefront 


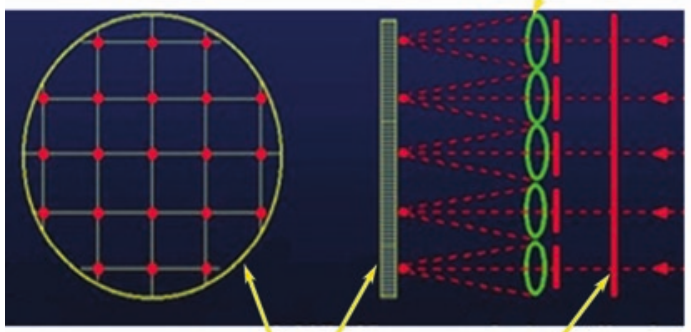

Video sensor Perfect wavefront

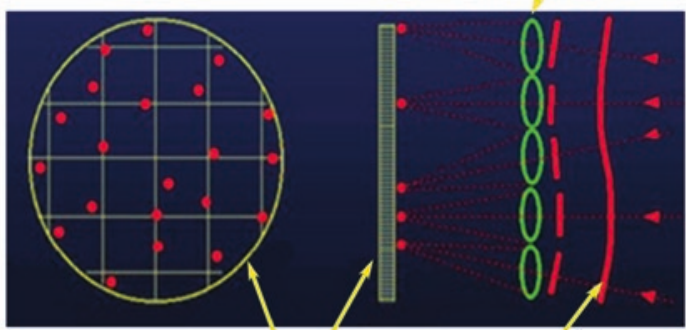

Video sensor Aberrated wavefront

Fig. 16.4 Left: Incident plane wave resulting in a square grid of spots. Right: Distorted wavefront causes lateral displacement of spots
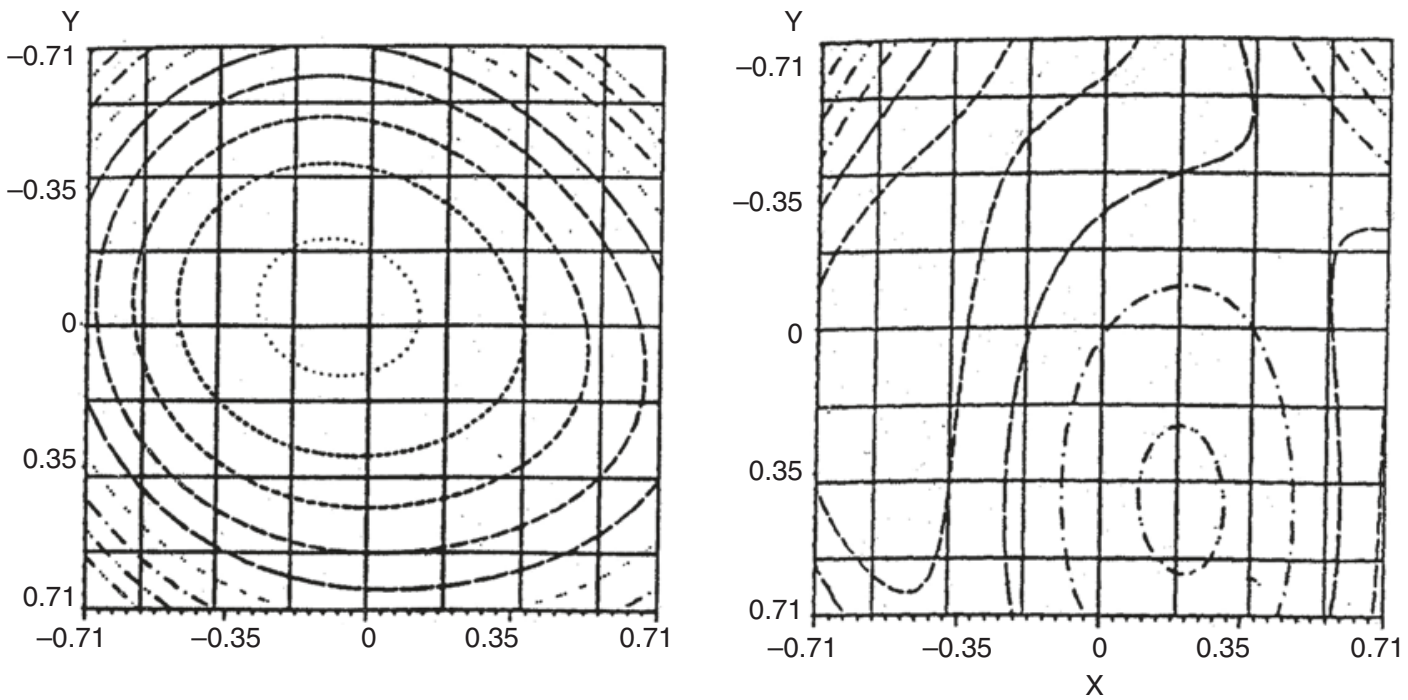

Fig. 16.5 Equal level contour map of a human eye. Left: Overall wavefront, Right: Higher order aberrations (third- and fourth-order Zernike's) (adapted by permission from Optical Society of America: [6])
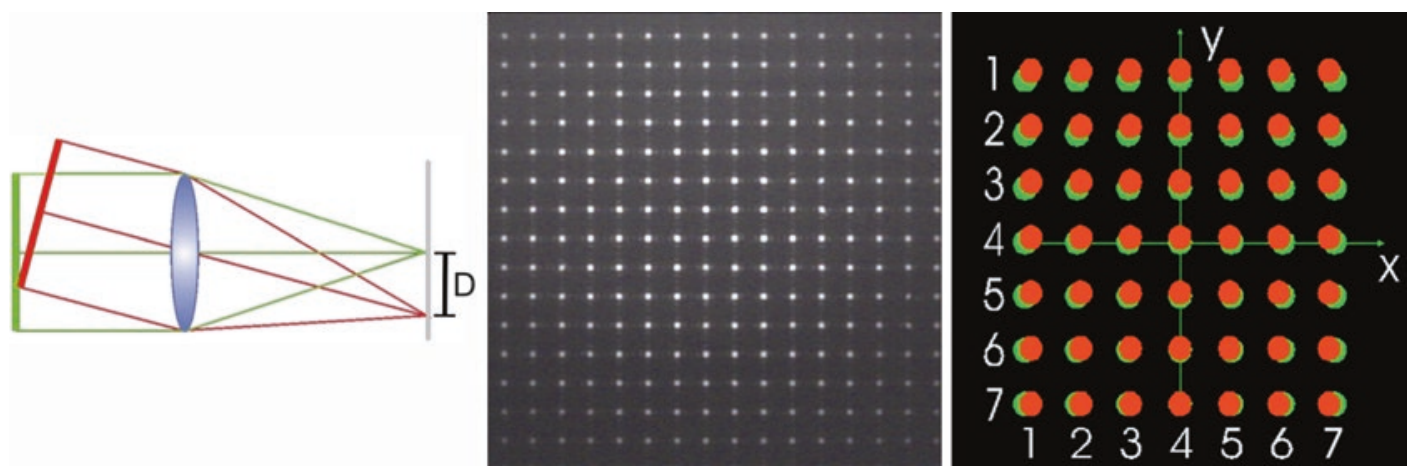

Fig. 16.6 Principle of WaveScan ${ }^{\mathrm{TM}}$ measurements. Ideal wavefront represented as a regular grid spots coded with green color. Distorted wavefront given by an irregular grid of spots coded with red color 
is zero, too. The RMS is thus simply the mean squared value of the wavefront over the pupil.

$$
\begin{aligned}
R M S & =\sqrt{\frac{\int_{0}^{r} \int_{0}^{2 \pi} W(\rho, \theta)^{2} \rho d \rho d \theta}{\int_{0}^{1} \int_{0}^{2 \pi} \rho d \rho d \theta}} \\
& =\sqrt{\frac{1}{\pi} \int_{0}^{12 \pi} \int_{0}^{12} W(\rho, \theta)^{2} \rho d \rho d \theta}
\end{aligned}
$$

In taking mean values of the Zernike polynomials the integral can be replaced by a sum of the weighted coefficients. For a real pupil size, the integration will be from 0 to $\mathrm{r}$.

$$
\begin{gathered}
R M S=\sqrt{\frac{\int_{0}^{r} \int_{0}^{2 \pi} W(\rho, \theta)^{2} \rho d \rho d \theta}{\int_{0}^{r} \int_{0}^{2 \pi}\left(\sum_{i=0}^{\text {order }} c_{i} Z_{i}(\rho, \theta)\right)^{2} \rho d \rho d \theta}} \\
=\sqrt{\frac{1}{\pi r^{2}} \sum_{i=0}^{\text {order }} c_{i}^{2} \int_{0}^{2 \pi} \int_{0}^{1} Z_{i}^{2}(\rho, \theta) \rho d \rho d \theta} \\
=\sqrt{\frac{1}{\pi r^{2}} \sum_{i=0}^{\text {order }} c_{i}^{2} Z_{i}^{\prime 2}}
\end{gathered}
$$

With $Z_{i}^{\prime 2}=$ weighting coefficient for each Zernike. It depends from the radial and angular order.

$Z_{i}^{\prime 2}=\frac{1}{\left(2-\delta_{l}\right) *(n+1)}$ with $i=\frac{n(n+1)}{2}+\frac{n-l}{2}+1$

The RMS can be calculated simply as a root of the sum of coefficients. The peak to valley (PTV) is closely connected to the RMS. While the PTV depends heavily on just two extreme values, the RMS is a kind of mean value received from the complete set of data points. This makes the RMS much more stable against deviations.

\subsubsection{Optical Aberration Index (OAI).}

The OAI is defined as

$$
O A I=1-e^{(-R M S)}
$$

The OAI has values between zero and one. Zero stands for an optical system that is perfect and 1 for infinite aberrations. The RMS-value is given as a fraction of the wavelength of light. The OAI is very sensitive in the typical range for higher order aberrations. It was introduced as an even simpler scale for the optical quality of an eye.

\subsubsection{Modulation Transfer Function (MTF).}

A typical target for testing the quality of an optical system consists of a series of alternating black and white bars of equal width with a contrast of 1 . These targets are connected to a Snellen E chart, as used in ophthalmology. The MTF gives the contrast of the image (as a percentage of the contrast of the object) in dependence of the frequency. The contrast is defined by:

$$
\text { Contrast }=\frac{I_{\max }-I_{\min }}{I_{\max }+I_{\min }}
$$

The MTF may be compared to the aerial image modulation (AIM) curve. This curve shows the smallest amount of modulation a sensor like a CCD camera or the retina is able to detect. The AIM is a function of the frequency used as well. As the MTF normally goes down with frequency increasing, the AIM increases with frequency. The point of intersection gives the resolution.

$$
\operatorname{MTF}(v)=\frac{M_{i}}{M_{o}}=\frac{2}{\pi}(\Phi-\cos \Phi \sin \Phi)
$$

With

$$
\Phi=\arccos \frac{\lambda v}{2 N A}
$$

$$
v=\text { frequency in } \frac{\text { cycles }}{\mathrm{mm}}
$$

$N A=$ numerical aperture

$$
\lambda=\text { wavelength }
$$




\subsubsection{Point Spread Function (PSF)}

The point response of an optic should still be a point. Even if the optic is perfect the response is a pattern due to diffraction. In a real system, the aberrations widen the image up to a spot. The spot is represented by a two-dimensional distribution. This is described by the PSF.

If the aberrations are smaller than $0.25 \lambda$ (Rayleigh criterion) the diffraction pattern provides a good description of the PSF. Up to about $2 \lambda$, it is appropriate to consider the manner in which the aberration affects the diffraction pattern. For larger wavefront aberrations illumination described by ray tracing is sufficient for description.

\subsubsection{Application of the Performance Indices in a Normal Human Eye}

In Fig. 16.7, the different performance indices are presented for a normal human eye. On the left part, a color-coded presentation of the wavefront is shown. The RMS of $0.23 \mu \mathrm{m}$ results in an OAI of 0.205 . On the middle part, the MTF is plotted, as well as the diffraction-limited MTF for a $6 \mathrm{~mm}$ pupil. On the right part, the PSF is graphically presented.

\subsection{Principle of Closed-Loop Adaptive Optical Control}

In Fig. 16.8, the principle of closed-loop adaptive optical control is schematically demonstrated. The wavefront of light which is distorted due to optical aberrations of the optical system, e.g. the human eye, is measured by a wavefront sensor. The reconstructed wavefront is dithered on a wavefront controller, e.g. an active mirror in order to compensate for the optical aberrations. Thus, through an aberrating medium, an aberration-free optical image can be achieved.
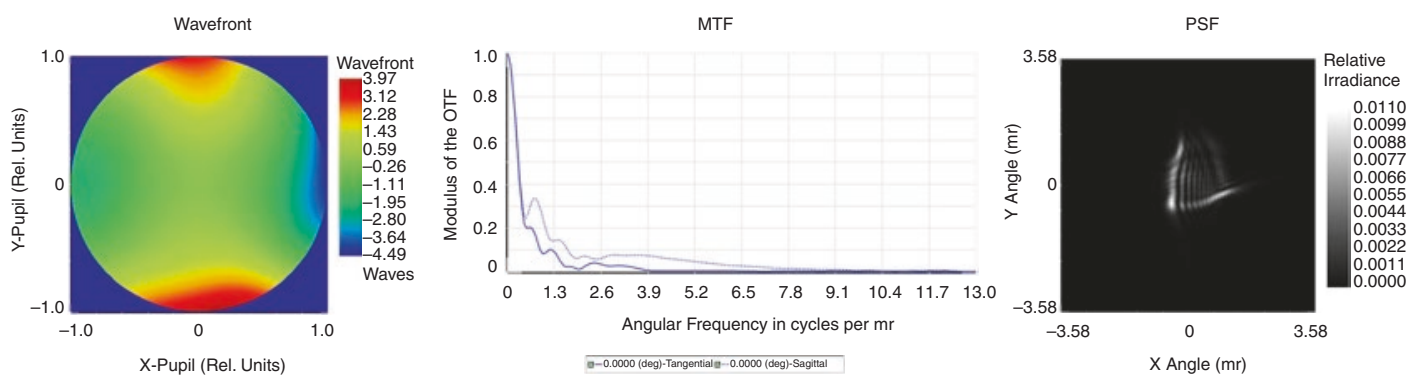

Fig. 16.7 Different representations of image quality of human eye

Fig. 16.8 Principle of closed-loop adaptive optical control

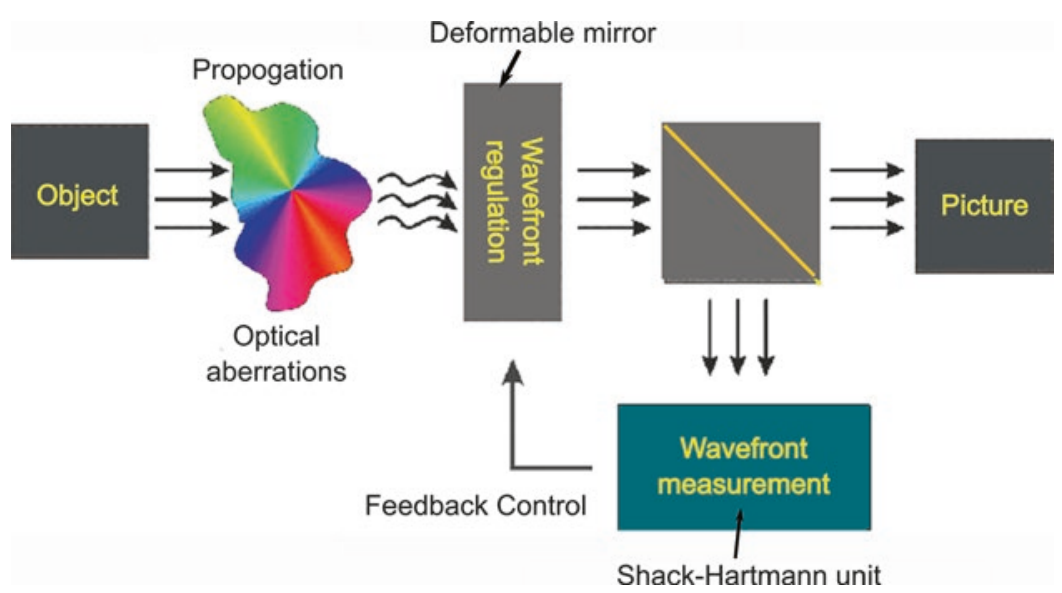


Calar Alto, 8 Dec.1997

$\mathrm{BD}+31643$ images taken with Omega-Cass in K-band

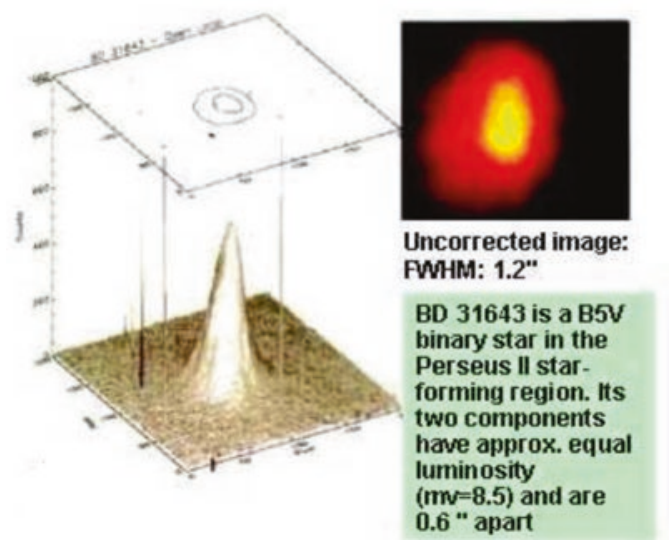

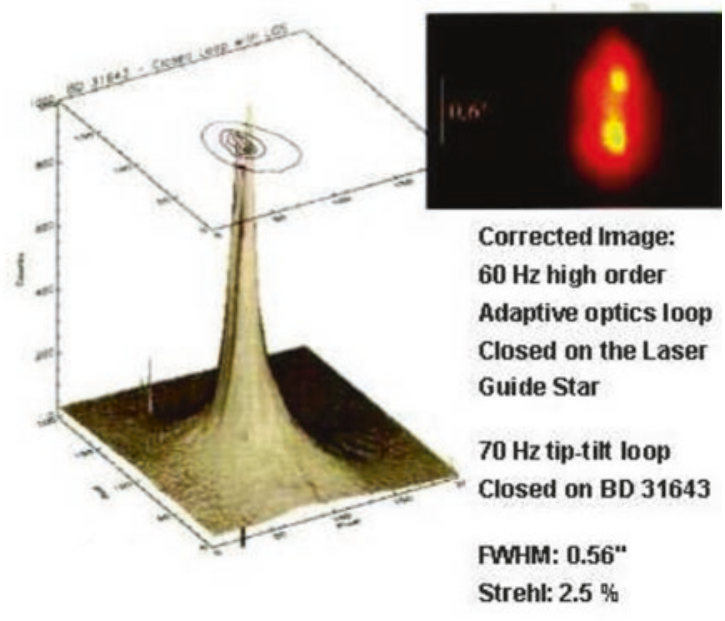

Fig. 16.9 Adaptive optics in astronomy: (left) speckle pattern, (right) sharpened picture

\subsubsection{Adaptive Optics in Astronomy}

Wavefront technology and closed-loop adaptive optical control were originally developed for astronomical applications. It was used to measure wavefront distortions that occurred when light traveling through the atmosphere entered an optical telescope. By applying closed-loop adaptive optical controls the speckle patterns of the star images could be improved towards diffractionlimited performance. In Fig. 16.9, the principle of operation of an adaptive optical closed-loop system on an optical telescope is demonstrated. In the left image the speckle pattern of an astronomical object, aberrated by the turbulent atmosphere is shown. In the right image, the sharpened picture after engagement of the closed-loop adaptive optical control demonstrating a double star image at high spatial resolution is depicted. The measurements were performed at the Calar Alto Optical Telescope operated by the Max Planck Institute for Astronomy, Heidelberg [15].

\subsubsection{History of Adaptive Optics at the University of Heidelberg}

In Fig. 16.10 a number of active mirrors and wavefront sensors developed at the Kirchhoff
Institute of Physics, University of Heidelberg in the last 40 years are depicted. The first generation foil mirror was successfully applied for the real-time compensation of aberrations of the human eye for high-resolution imaging of the retina [5]. In 2002, closed-loop operational results of the second generation foil mirror are reported [16]. In early 2000s a multi-segment microchip mirror was developed, exhibiting approximately 100,000 mirror facets, each able to slightly shift the phase of a local component of the wavefront in order to compensate for the detected wavefront error. In the lower part of Fig. 16.10 two different realizations of ShackHartmann wavefront sensors are shown. On the left-hand side, a cylindrical lens array with CCD detector is photographed which was applied for the first time to measure the aberration of the human eye in real time [6]. On the right-hand side, a custom ASIC chip detector which is used in combination with a custom micro-lens array is shown [17]. The ASIC chip is divided into a matrix of clusters, consisting of photodetectors and signal processing circuitry. By analogous signal processing in winner-takes-all circuitry, the highest photocurrent is detected and position is calculated. The data obtained are evaluated in real time for reconstruction of the wavefront of the light. 
Fig. 16.10 History of adaptive optical elements, as developed at the University of Heidelberg

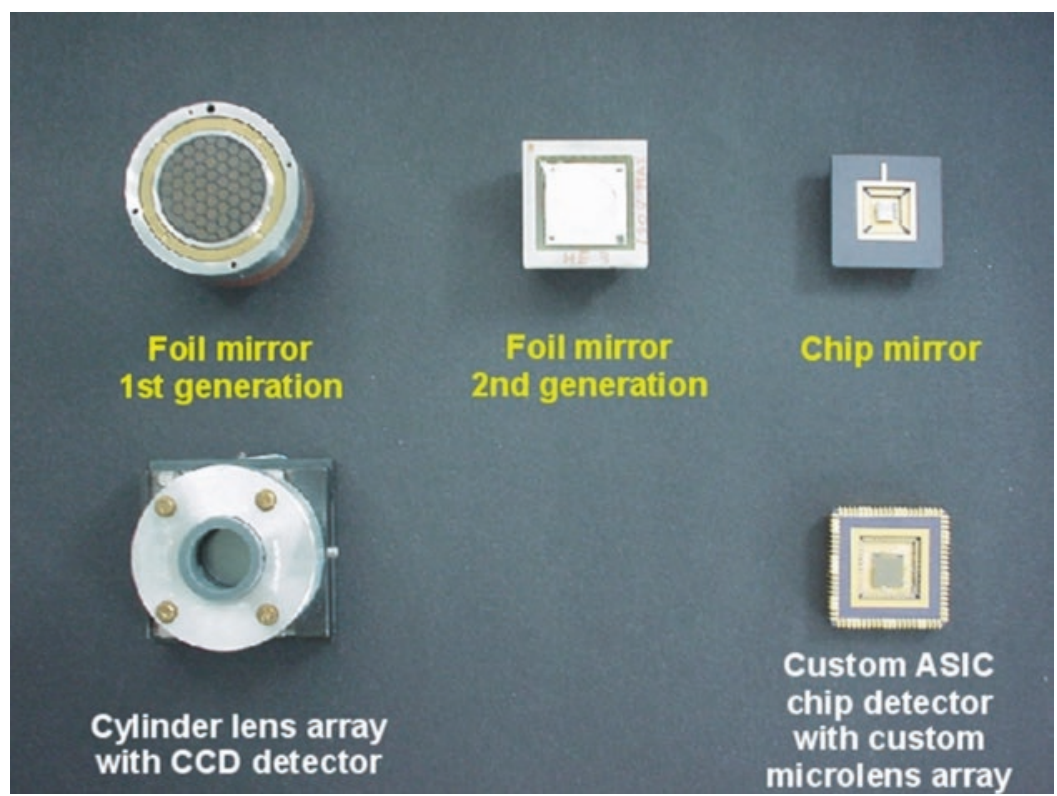

\subsection{Demonstration of Adaptive Optics Aberrometer}

An active matrix mirror is used in the device (see Fig. 16.11). It is an array of $200 \times 240$ micromirrors $(40 \mu \mathrm{m} \times 40 \mu \mathrm{m}$ each). Each one of the mirrors can be lowered up to $400 \mathrm{~nm}$ independently. The mirrors can only be lowered without the facility of tilting. With this technique, wavefronts can be corrected up to the double height of deflection-more than one wavelength. By using the $2 \pi$-phase wrapping method (Fig. 16.12) the range of the wavefront deformations to be corrected can be enlarged by far. The $2 \pi$-phase wrapping method makes use of the phase properties of light. Sag of $2 \pi$ between two neighboring mirrors has no effect on the direction of the light and can be subtracted without any effect on the wavefront. So, the range of movement needed for the correction of any wavefront-deformation can be reduced to $\lambda / 2$. In fact, the use of the mirror is limited to the light of one wavelength when using the $2 \pi$-phase wrapping method [16].
For an objective test of the active mirror, a test device was constructed (Fig. 16.13) and this device allows to measure the phase plate and look through it into the instrument at the same time. From the camera, at the test device, an image of the target is obtained. For the measurements presented here, a target was used with $1^{\prime}$ apex angle, corresponding to a Visual Acuity (VA) of 1.0.

In Fig. 16.14, the corrections of phase plates are depicted. The wavefront map, the PSF and the real images of the Siemens-star targets are shown, respectively. On the left-hand side, a phase plate with fourth order spherical aberration is evaluated, on the right-hand side; a phase plate with third order trefoil aberration is imaged.

In Fig. 16.15, case studies of patients are exemplified. For patient JS (OD) an improvement of the RMS values from $0.397 \mu \mathrm{m}$ to 0.149 is achieved, for patient RB (OD) the RMS value changes from $0.336 \mu \mathrm{m}$ to $0.113 \mu \mathrm{m}$. The patients gain one line on the Snellen chart, from VA 20/16 to VA 20/12.5, and from VA 20/20 to VA 20/16, respectively. 
Fig. 16.11 Setup of microchip mirror
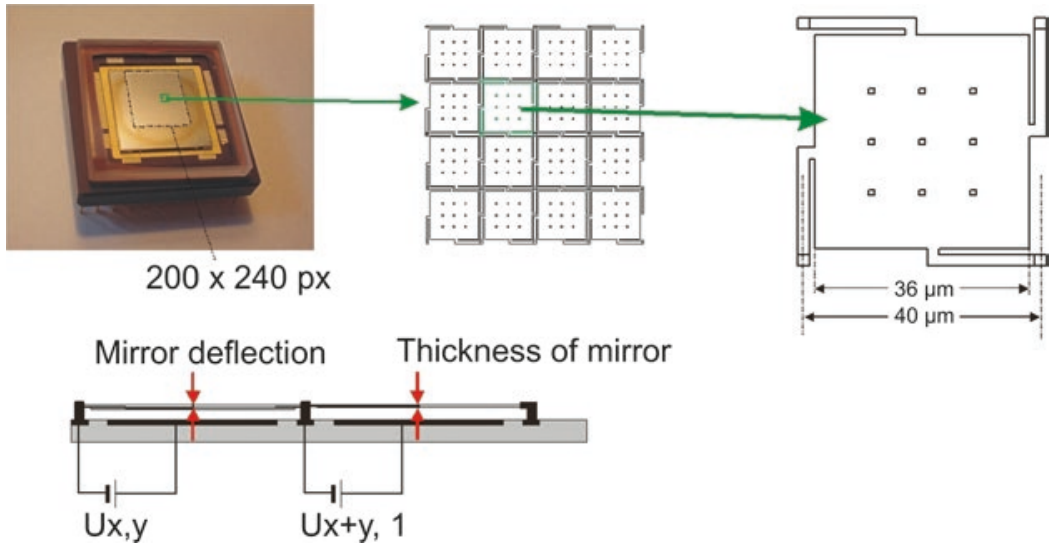

Fig. $16.122 \pi$-phase wrapping control of the micro mirror

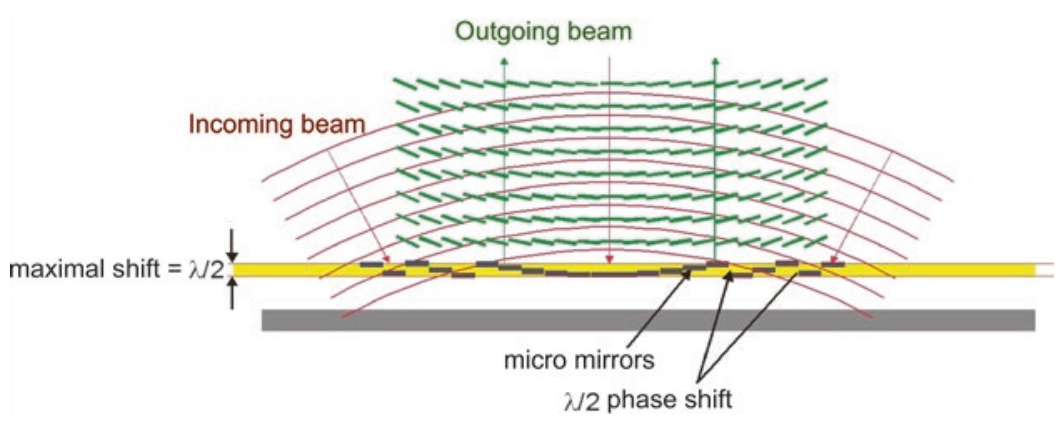

$\lambda / 2$ phase shift

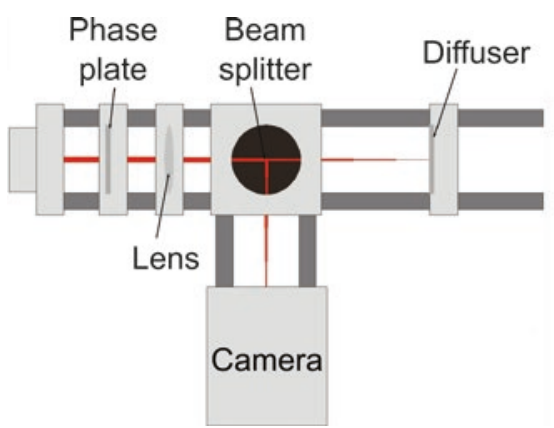

Fig. 16.13 Left: Test device for the active mirror. Light entering from the left through the phase plate is divided by the dichroic beam splitter cube. The aberrations get mea- sured in the right arm. The bottom arm is used to record an image of the target. Middle and Right: Visual Acuity Chart ous orders, from second to sixth order, are plotted. The effect on RMS total (brown graph) amounts to an improvement by a factor of two, from RMS $0.3 \mu \mathrm{m}$ to RMS $0.15 \mu \mathrm{m}$.
In Fig. 16.16, the reduction of higher-order RMS values of human eyes with adaptive optics RMS values of human eyes with adaptive optic compensation is visualized. The effects on vari-

\subsubsection{Clinical Prototype Adaptive Optics Aberrometer}

In Fig. 16.17, an adaptive optics aberrometer is depicted, with which the first clinical studies on adaptive optics vision testing were performed,

\section{E I Ш E $ヨ$}

E $\exists \boldsymbol{m} \amalg \exists$

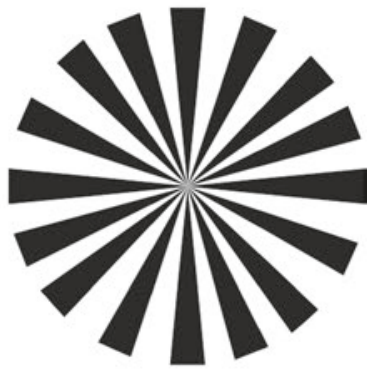


based on adaptive optics technology [18]. The study was performed at eye clinics near Würzburg, Germany. The reproducibility and accuracy of the measurements were assessed. Through adaptive optics compensation, the aberrations of higher orders, like e.g. coma and spherical aberration could be reduced from
$0.3 \mu \mathrm{m}$ to $0.1 \mu \mathrm{m}$ RMS error. The limit of resolution of the instrument was less than $0.1 \mu \mathrm{m}$, corresponding to a measurement accuracy of $10^{-5}$, considering the length of the eye of approximately $20 \mathrm{~mm}$, achieved on living eyes. As an interesting result, we found that approx. Half of the patient population exhibited an amount of

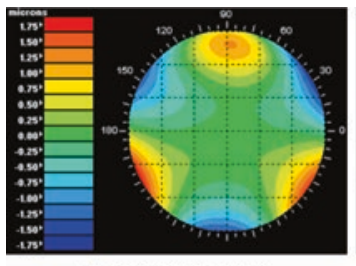

uncorrected RMS: 0.54 um

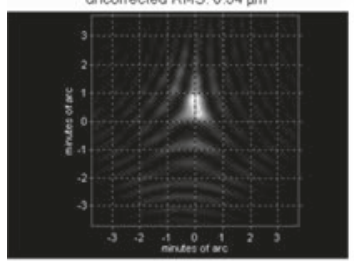

uncorrected effective blur: $1.66 \mathrm{dpt}$

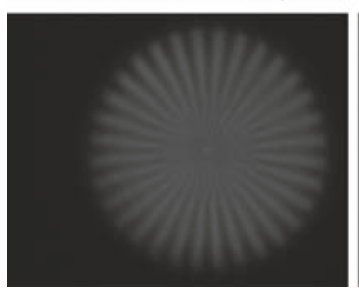

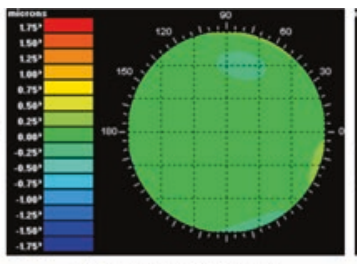

best corrected RMS: 0.06 um

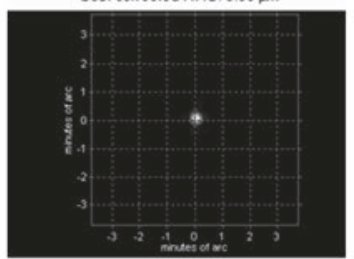

best corrected effective blur. $0.17 \mathrm{dpt}$

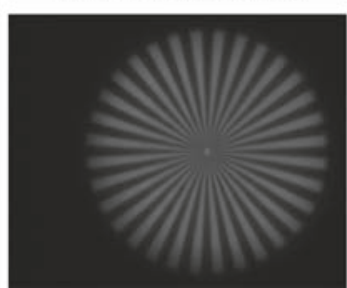

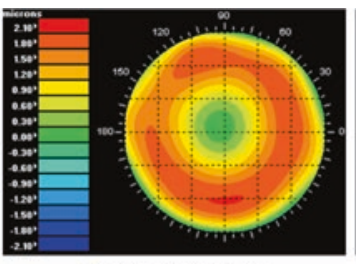

uncorrected RMS: 0.54 um

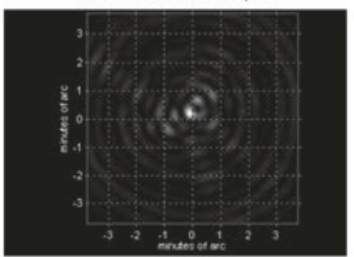

uncorrected effective blur: $1.67 \mathrm{dpt}$

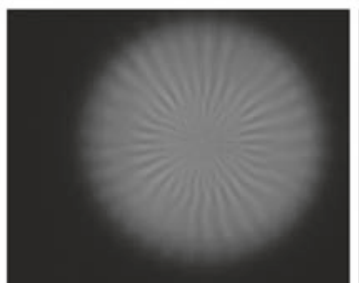

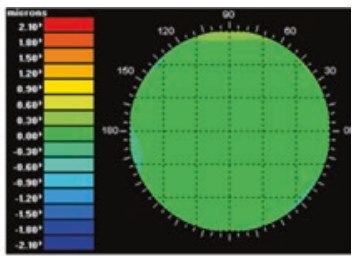

best corrected RMS: 0.07 um

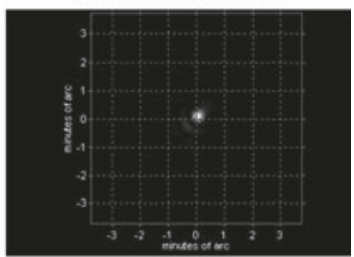

best corrected effective blur: $0.22 \mathrm{dpt}$

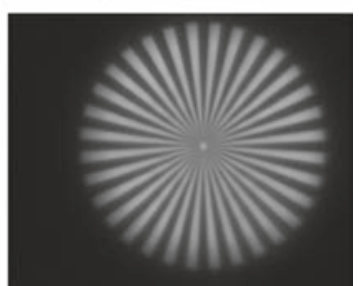

Fig. 16.14 Left: Correction of phase plates of fourth order spherical aberration. Right: Correction of phase plates of third order trefoil aberration. WF wavefront map, PSF point spread function, Image real image

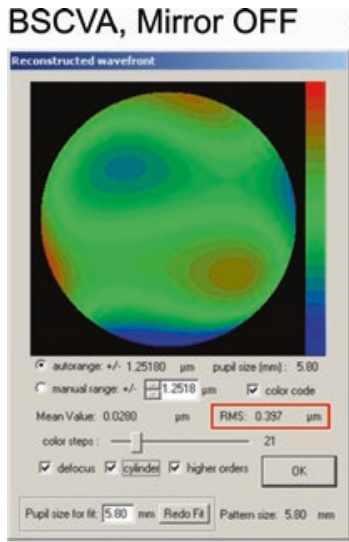

VA 20/16

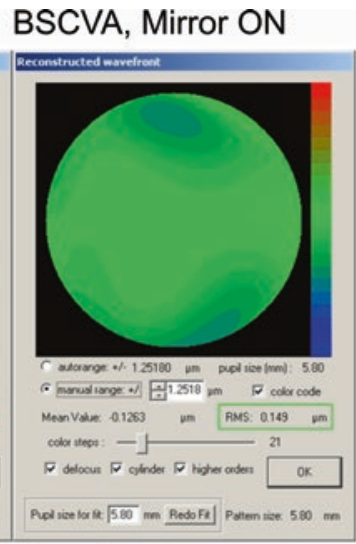

VA $20 / 12.5$

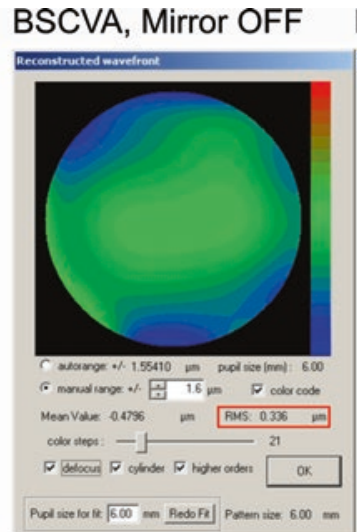

VA $20 / 20$
BSCVA, Mirror ON

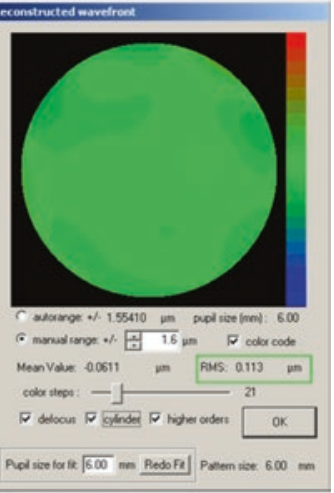

VA $20 / 16$

Fig. 16.15 Left: Case study—Patient JS (OD). Right: Case study—Patient RB (OD) 


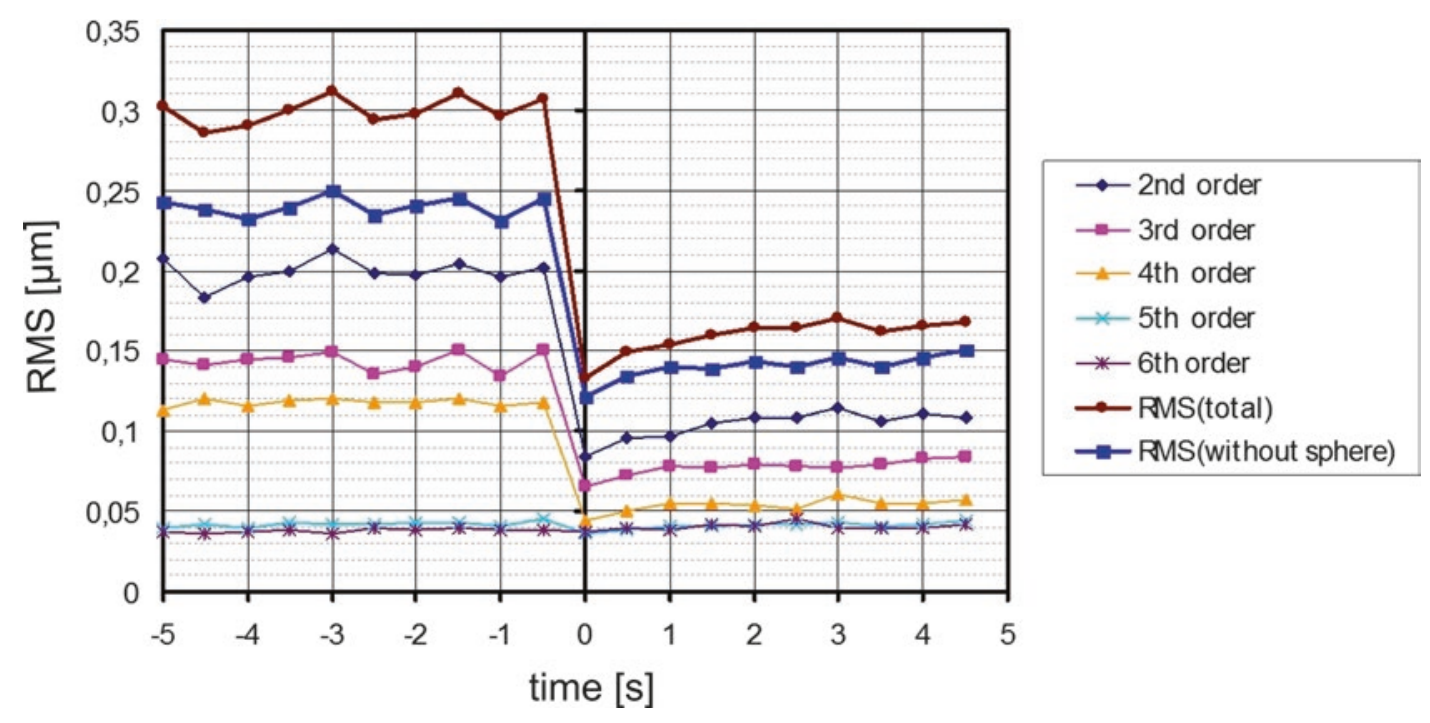

Fig. 16.16 Higher-order aberration reduction in human eyes with the microchip mirror

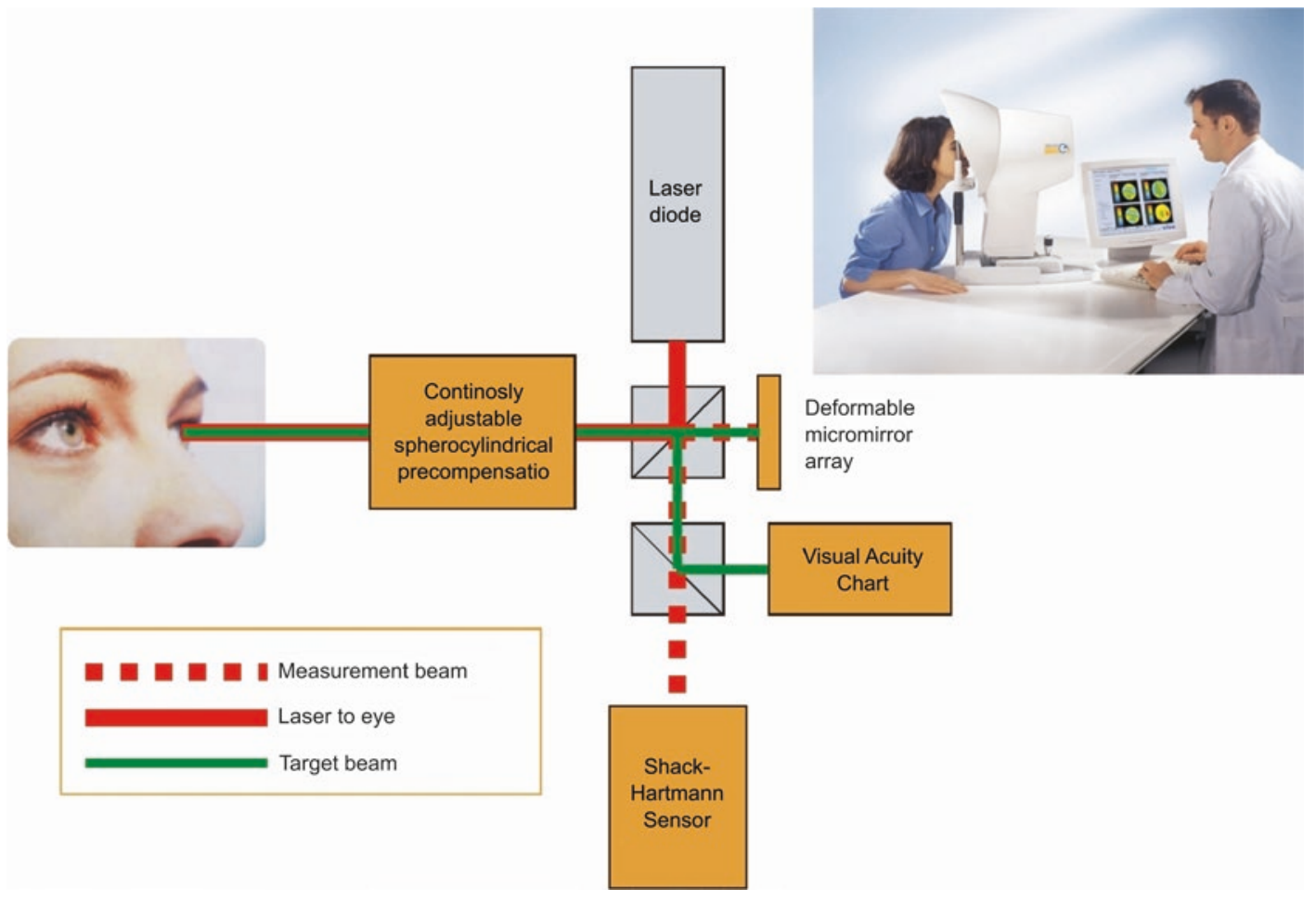

Fig. 16.17 Setup of adaptive optics aberrometer 
$0.3 \mu \mathrm{m}$ of higher-order optical aberrations of the eye, which play a considerable role with regard to visual acuity, for the whole human population, and which cannot be corrected with conventional eyeglasses or contact lenses.

\subsubsection{A Case Study on a Refractive- Surgical Patient with Clinical Prototype}

In Figs. 16.18 and 16.19 the correction of higherorder wave aberrations for a refractive surgical patient is demonstrated. In Fig. 16.18 on the right- hand side, the uncompensated coma modeled into a phase plate is shown, resembling the aberration of a human eye before therapeutic custom ablation correction. The PTV difference amounts to $2 \mu \mathrm{m}$, the RMS error to $0.72 \mu \mathrm{m}$. The patient achieved the best spectacle-corrected visual acuity (BSCVA) of 20/40. On the left-hand side of Fig. 16.18 the appearance of the WaveScan ${ }^{\mathrm{TM}}$ tunnel target is blurred, correspondingly. In Fig. 16.19, the compensated wavefront and the target image are depicted. By closed-loop adaptive optical control, the RMS error can be reduced to $0.07 \mu \mathrm{m}$, corresponding to one-tenth of a wavelength of light. The WaveScan ${ }^{\mathrm{TM}}$ tunnel target image is sharp-
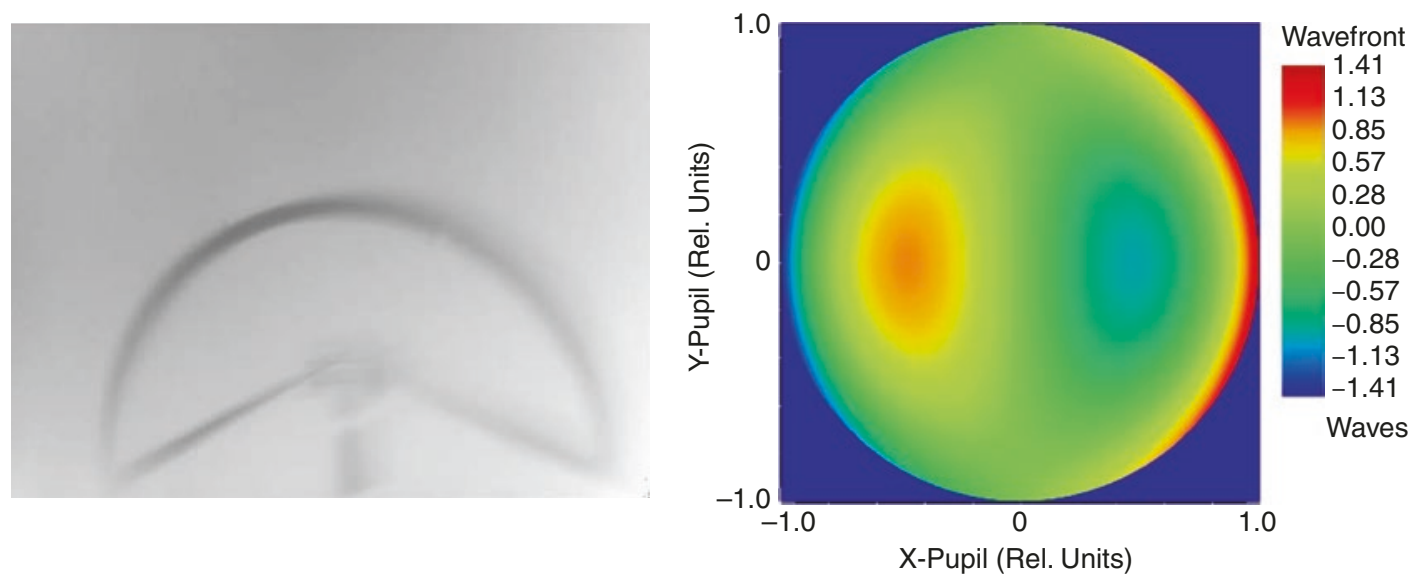

Fig. 16.18 Phase plate simulating human eye with high coma. Left: defocused image, Right: uncompensated wavefront
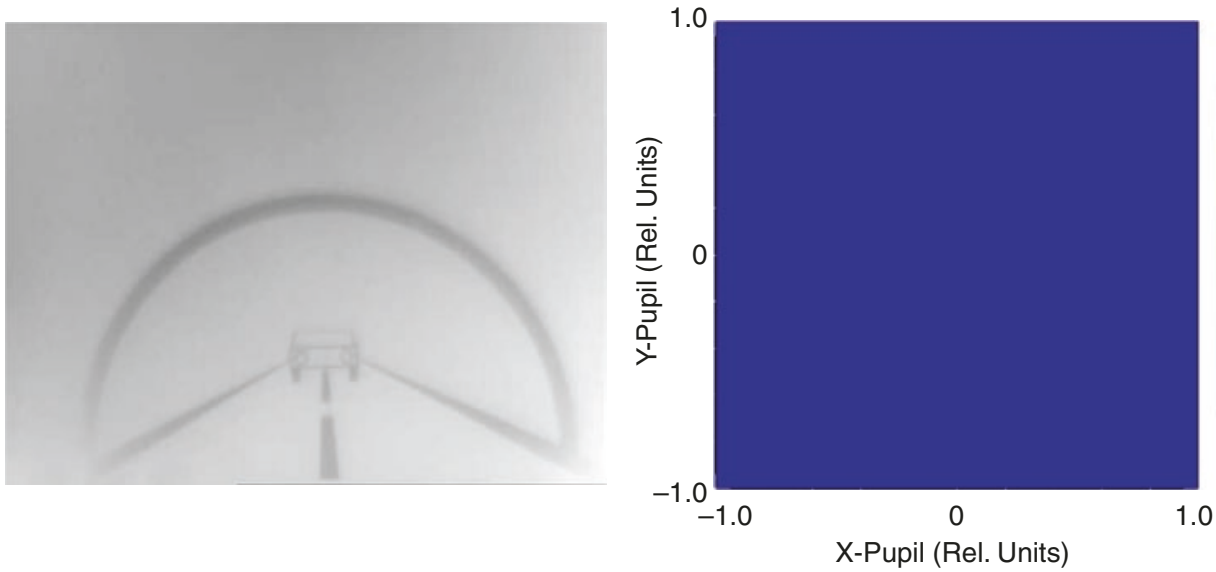

Wavefront $1.00 \mathrm{E}-005$ 9.00E-006 $8.00 \mathrm{E}-006$ 7.00E-006 6.00E-006 $5.00 \mathrm{E}-006$ 4.00E-006 3.00E-006 2.00E-006 $1.00 \mathrm{E}-006$ $0.00 \mathrm{E}+000$ Waves

Fig. 16.19 Compensation of high coma aberration. Left: Focused image, Right: compensated wavefront 
ened, accordingly. Indeed, the patient's vision was improved by a therapeutic custom ablation procedure to nearly perfect 20/12.5 performance.

\subsection{The Limits of Human Vision}

In Fig. 16.20, the results of the wavefront measurements for a left eye (OS: "Oculus Sinistrus", left eye) of patient TK (OS) are depicted. A modest amount of coma, a third order aberration, is present. The RMS error of the higher-order aberrations of this eye amounts to $0.656 \mu \mathrm{m}$. About $20 \%$ of all human eyes exhibit such an RMS error. For older patients, this percentage is substantially higher, measured at a pupil size of approximately $6 \mathrm{~mm}$ in diameter. After engaging the micro-mirror device, i.e. with adaptive optics compensation, the RMS error can be reduced to $0.166 \mu \mathrm{m}$, corresponding to approximately $\lambda / 5$. At the same time, the patient's visual acuity is enhanced. In this case, the visual acuity improves from $20 / 20$, i.e. normal vision, to $20 / 16$, i.e. the patient can see one additional line on the Snellen chart, i.e. the 20/16 line.

In Fig. 16.21, research results are shown, which exemplify the limits of human vision. The MTF is plotted in dependence of spatial frequency, measured as line pairs, respectively cycles, per degree of visual angle, i.e. cycles/ degree, visualizing the optical quality of a human eye. Regarding the MTF characteristic, a spatial frequency of 30 cycles/degree corresponds to $20 / 20$ vision, corresponding to a smallest resolvable visual angle of 1 arcminute. A smallest resolvable visual angle of 0.5 arcminute results in $20 / 10$ vision, corresponding to a spatial frequency of 60 cycles per degree. For reference, the diffraction-limited MTF is sketched for a $3 \mathrm{~mm}$ pupil (blue color) and a $6 \mathrm{~mm}$ pupil (black color). The curve, depicted by the red broken line, characterizes the MTF of the left eye of patient TK (OS), uncompensated. With a $6 \mathrm{~mm}$ pupil, patient TK (OS) exhibits an MTF of 0.15 at 30 cycles/ degree, corresponding to $20 / 20$ vision. A human eye needs a contrast of approximately $10 \%$, in order to perceive a fine grid of lines, spaced at the related spatial frequency. After adaptive optics compensation, the green-broken MTF curve results, with an improvement of the image contrast by a factor of 5 at 30 cycles/degree. As described previously (see Fig. 16.20), patient TK (OS) reached 20/16 vision, after adaptive optics compensation. In the case of patient WE (OD), (OD: Oculus Dextrus, i.e. right eye), (red solid line) only minute higher-order aberrations are present; nevertheless the adaptive optics compensation
Fig. 16.20 Case study: Patient TK (OS)

\section{BSCVA, Mirror OFF}

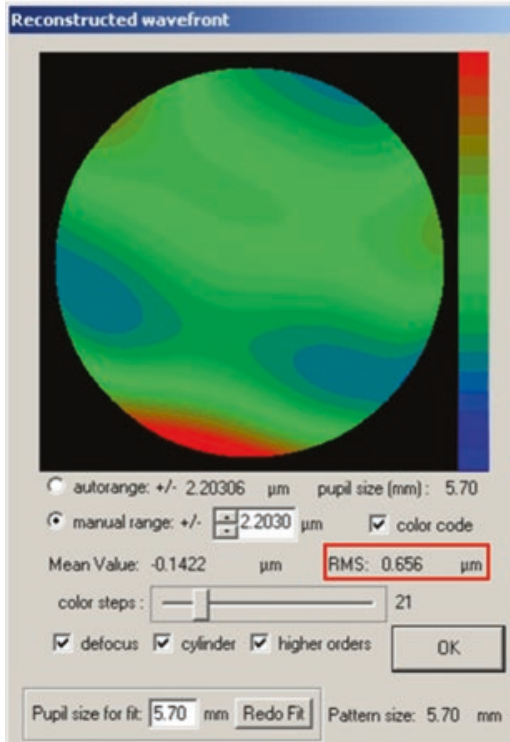

BSCVA, Mirror ON

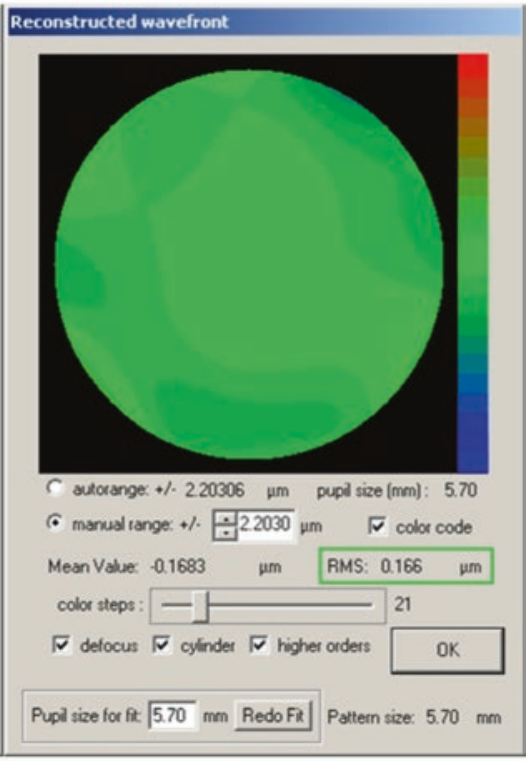

VA $20 / 16$ 


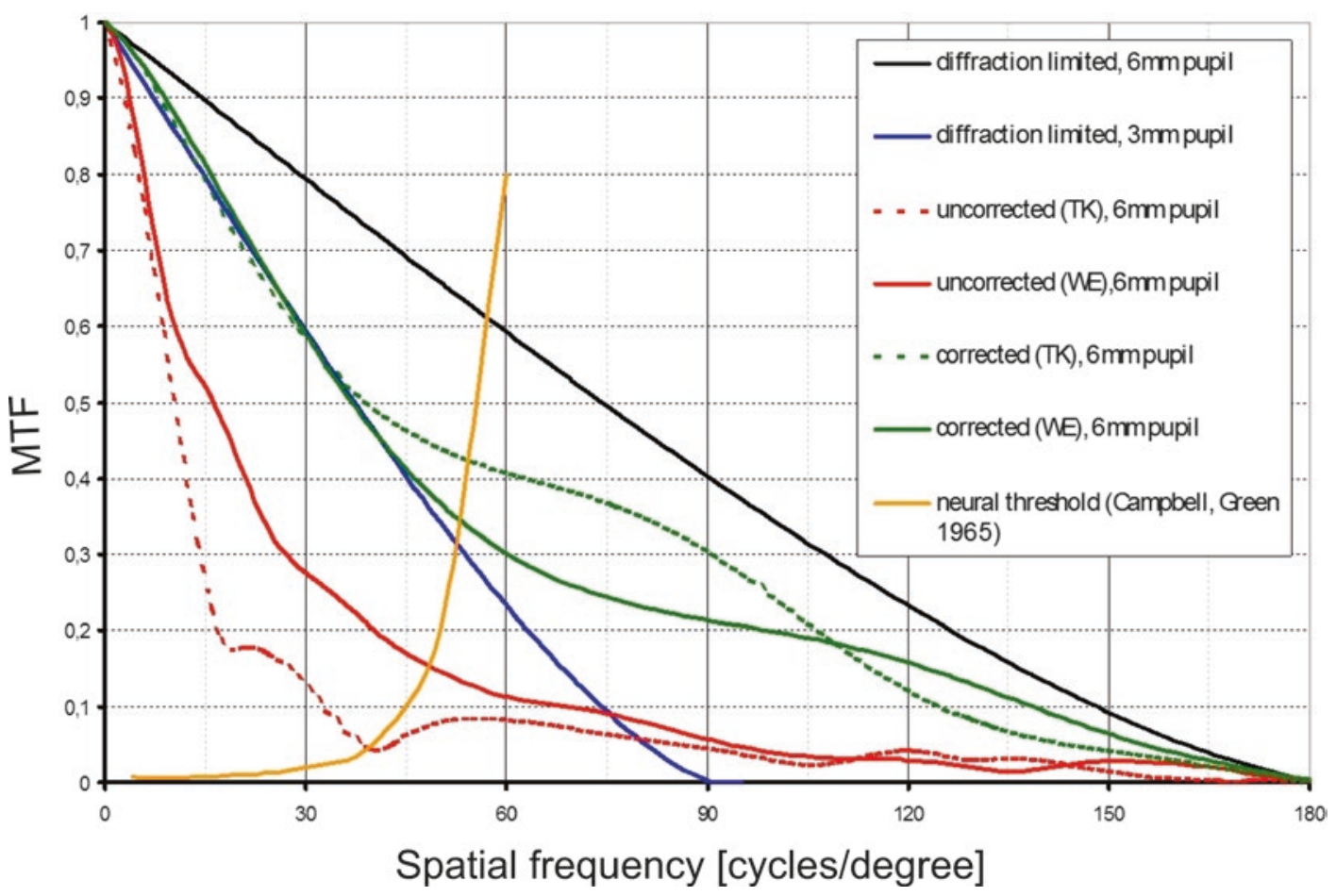

Fig. 16.21 Visual acuity, MTF

results in a considerable amount of improvement of the MTF, as such that the value of the MTF stays above MTF $=0.1$ even at 120 cycles/ degree, corresponding to $20 / 5$ vision, amounting to four times normal human vision. For patient WE (OD), uncorrected vision amounted to 20/16, and reached 20/10, after compensation. Human eyes can reach at maximum 20/10 vision, limited by the neuronal threshold of visual acuity.

Campbell and Green published a seminal paper on the neuronal limit of human vision in 1965 [19]. The neuronal limit of human vision is related to the size of the retinal photoreceptors. In the fovea, the retina exhibits cones with smallest dimensions of $2.2 \mu \mathrm{m}$, corresponding to a visual angle of 0.5 arcminutes, i.e. visual acuity of 20/10. At the limit of resolution, the lateral inhibition is disabled, i.e. the initial neuronal processing of the retina is rendered ineffective. An individual neuronal sensitivity curve is sketched in Fig. 16.21 in yellow color. Related to this individual characteristic, a contrast of 0.8 would be necessary to achieve a 20/10 vision. A contrast of 0.8 at 60 cycles/degree is above the diffraction-limited characteristic. Thus, the achievable improvement of the visual acuity through adaptive optics compensation of the optical aberrations of the human eye is limited by the individual neuronal threshold. Our clinical studies have shown that the neuronal threshold characteristics can be enhanced by adaption, based on a lengthy learning process.

However, as demonstrated above (see Fig. 16.21, MTF characteristic of patient $\mathrm{WE}(\mathrm{OD}))$, the optical quality of human eyes can be optimized to a smallest PSF of approximately $1.25 \mu \mathrm{m}$ spot-size ('airy-disc'), which is essential for high-resolution imaging of the retina of the human eye (see Chap. 17).

\subsection{Aberration-Free Retinal Imaging}

Retinal imaging has been an integral part of every ophthalmic examination for early diagnosis and follow-up the retinal diseases. Fundus cameras, SLOs, and OCT systems provide a macroscopic 
view of the retina, however, these instruments lack in transverse resolution to reveal the microscopic structures of the retina. Improving the resolution of the retinal images has always been the concern for the researchers [20]. The main limitation is the pupil size, where the diffraction dominates the PSF in small pupil size and the aberrations in large pupil size [21]. Adaptive optics facilitated the retinal imaging techniques by improving the image quality. Adaptive optics was first applied in a fundus camera by Liang et al. in 1997 to resolve the cone photoreceptors [7]. Since then adaptive optics has been implemented by many researchers for aberration-free retinal imaging. In 2002, Burns et al. used phase plates with confocal SLO for higher-order aberrations and achieved a $26 \%$ increase in contrast of the retinal blood vessels
[22]. Meantime, Roorda et al. presented the first adaptive optics confocal scanning laser ophthalmoscope (cSLO) using a Shack-Hartmann sensor providing a real-time microscopic view of the human retina. The resolution achieved was $2.5 \mu \mathrm{m}$ lateral and $100 \mu \mathrm{m}$ axial compared to $5 \mu \mathrm{m}$ lateral and $300 \mu \mathrm{m}$ axial in conventional SLO [11]. Further, the OCT has also been integrated with adaptive optics for aberration-free retinal imaging to improve the axial and lateral resolution. Adaptive optics was integrated with ultrahigh resolution OCT and spectral domain OCT [10, 23].Likewise, the adaptive optics has been incorporated with TPO aimed at detecting the early onset of retinal diseases [9].

In Fig. 16.22, the adaptive optics improvement of the PSF through compensation of the
Fig. 16.22 Adaptive optics Improvement of PSF through compensation of the optical aberrations of a human eye. $W F$ wavefront map, $P S F$ point spread function
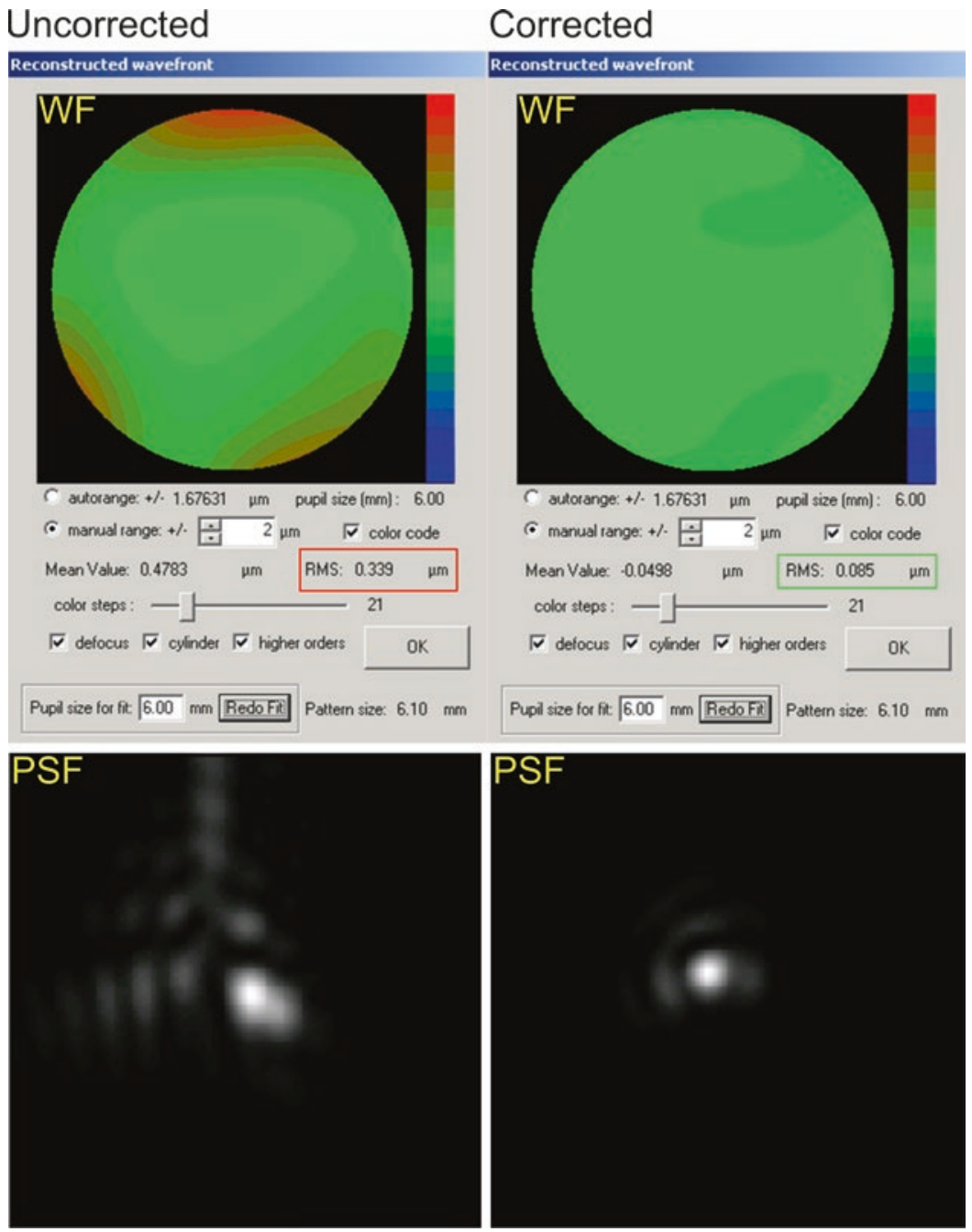


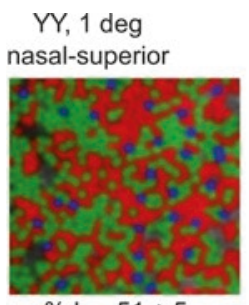

$\% \mathrm{~L}=51 \pm 5$

JC, 1.25 deg temporal

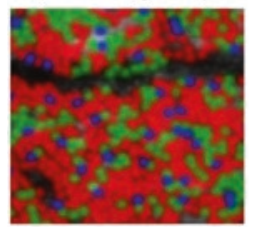

$\% \mathrm{~L}=66 \pm 4$

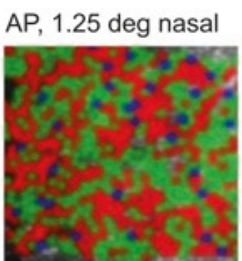

$\% \mathrm{~L}=51 \pm 3$

JW, 1 deg temporal

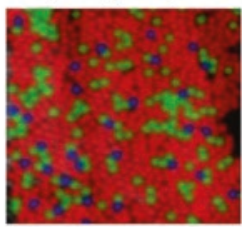

$\% \mathrm{~L}=80$

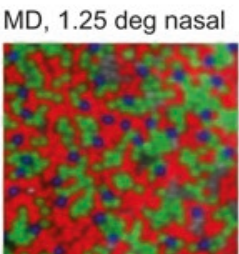

$\% \mathrm{~L}=60 \pm 3$

BS, 1.25 deg nasal

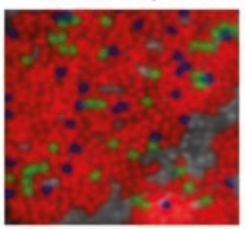

$\% \mathrm{~L}=94 \pm 3$
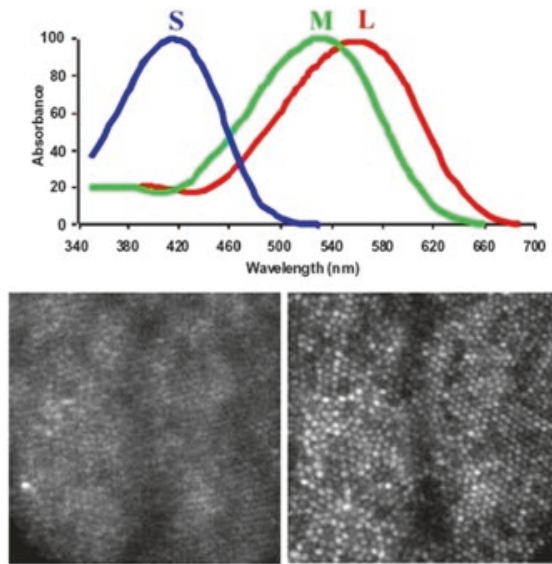

Without compensation

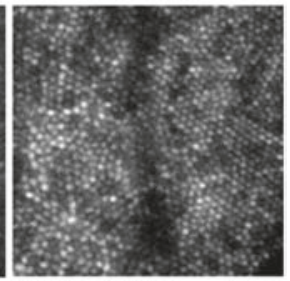

With Adaptive $\overline{5 \text { arcmir }}$ optics compensation

Fig. 16.23 The arrangement of the three cone classes (L/M-, S-cones) in the living human eye

higher-order optical aberrations of a human eye is demonstrated. The RMS wavefront error of the uncorrected wavefront was $0.339 \mu \mathrm{m}$, after compensation the RMS wavefront error of $0.085 \mu \mathrm{m}$ was measured. The uncorrected and corrected PSFs are depicted in the lower part of Fig. 16.22.

Human color vision depends on three classes of receptor, the short- (S), medium- (M), and long- (L) wavelength-sensitive cones. These cones are interleaved in a single mosaic so that, at each point on the retina, only a single class of cone samples the retinal image. As a consequence, observers with normal trichromatic color vision are necessarily color blind on a local spatial scale. The limit this places on vision depends on the relative numbers and arrangement of cones. Although the topography of human $\mathrm{S}$-cones is known, the human L- and M-cone sub mosaics have resisted analysis. Adaptive optics, a technique used to overcome blur in groundbased telescopes (see also Fig. 16.8), can also overcome blur in the eye, allowing the sharpest images ever taken of the living retina [24]. In Fig. 16.23, the arrangement of the three cone classes (L/M-, S-cones) in the living human eye are depicted. Adaptive optics and retinal densitometry are combined, to achieve aberration-free images of the living human retina. The proportion of $\mathrm{L}$ to $\mathrm{M}$ cones is strikingly different in two male subjects, each of whom has normal color vision. The mosaics of both subjects have large patches in which either M or L cones are missing. This arrangement reduces the eye's ability to recover color variations of high spatial frequency in the environment but may improve the recovery of luminance variations of high spatial frequency.

\subsection{Wavefront-Guided Laser Refractive Surgery (CustomVue)}

Wavefront-guided laser refractive surgery (CustomVue) was founded with a pilot study at the Augenpraxisklinik (Eye clinics) Heidelberg in the year 2000, performing the first fifty wavefront-guided eye corrections, world-wide. Subsequently, five hundred patients were treated in a multi-center FDA study in the United States.

In Fig. 16.24 the results of the FDA study are depicted. The blue bars of the bar diagram describe the visual acuity before surgery (BSCVA: "best spectacle-corrected visual acuity", see Fig. 16.24). All five hundred patients of the study group exhibited 20/20 vision, 59\% resp. $6 \%$ reached $20 / 16$ vision resp. 20/12.5 vision. The green bars of the bar diagram present the visual acuity after wavefront-guided refractive laser surgery (UCVA: uncorrected visual acuity (VA), see Fig. 16.24). After 

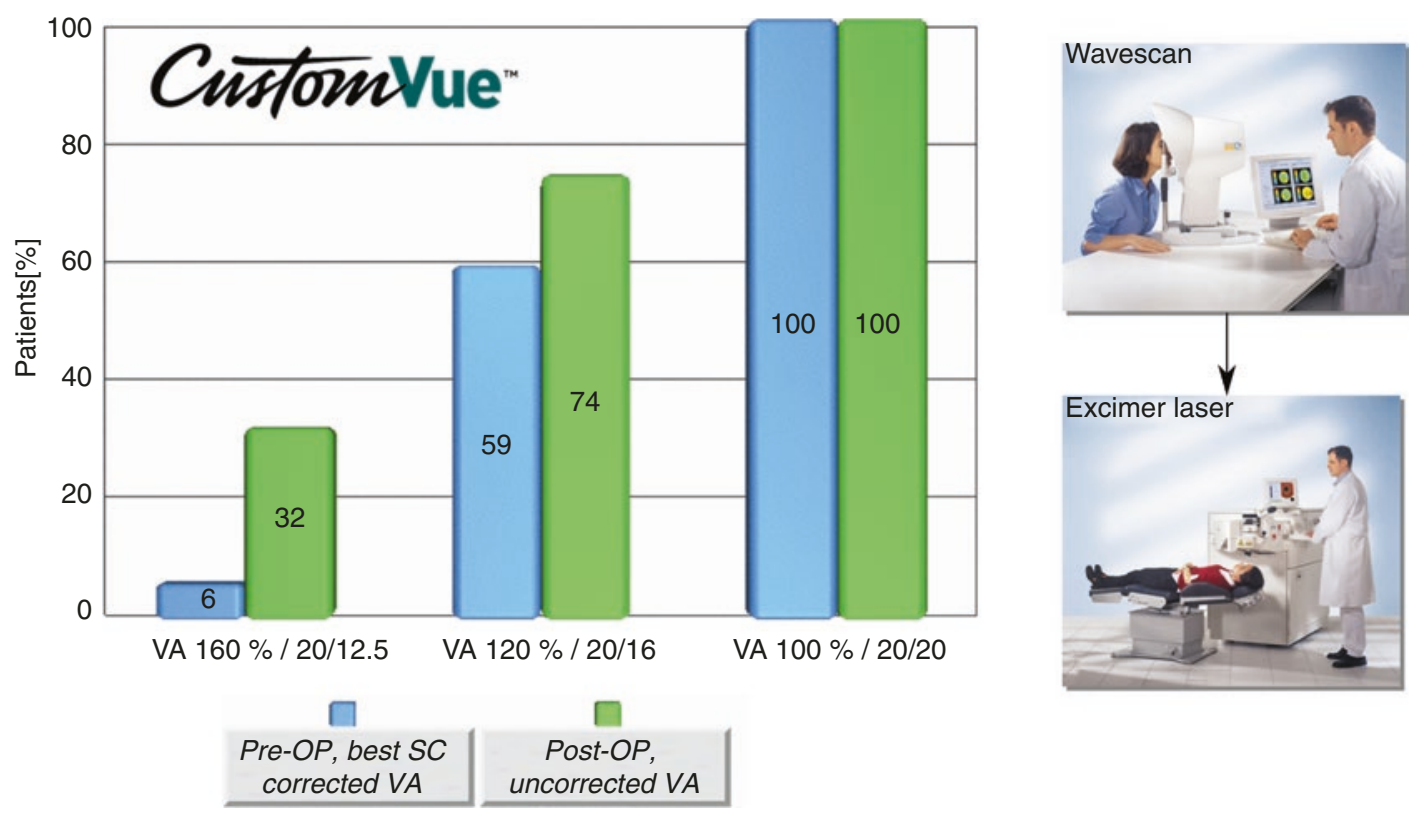

Fig. 16.24 Improving vision with custom refractive procedures

treatment, $100 \%$ of the patients maintained at least 20/20 vision, the number of patients with $20 / 16$ vision improved to $74 \%$, and the number of patients with $20 / 12.5$ vision reached $32 \%$, i.e. one hundred and fifty patients of the study group of five hundred patients reached the level of optically perfect vision. Since then, wavefront-guided laser refractive surgery has been successfully performed on many millions of patients.

\subsection{Summary}

The introduction of wavefront technology in ophthalmology allows determining the optical aberrations of the human eye, far beyond the sphero-cylindrical refractive error. Based on WaveScan technology the reproducibility and accuracy of the new technique were established in worldwide multicenter clinical studies, of which, one of the most powerful clinical applications is the wavefront-guided refractive surgery. In this chapter, it was demonstrated that closed-loop adaptive optical control allows for improved spatial resolution of aberration mea- surements, increasing the resolution limit by two orders of magnitude over, e.g. Shack-Hartmann technology. Also, adaptive optics has proven its ability to resolve the microstructures of the retina by correcting the optical aberrations down to diffraction-limit. The aberration-free retinal imaging with adaptive optics will improve our understanding of the visual system in the normal and diseased eye.

Acknowledgement The historical background and the basics of adaptive optics aberration measurements were published previously in reference [25].

\section{References}

1. Bille JF, Freischlad K, Jahn G, Merkle F. Image restauration by adaptive optical phase compensation. In Proceedings 6th international conference on pattern recognition, Munich, Germany. 1982.

2. Bille JF. US4579430A - Method and apparatus for forming an image of the ocular fundus. Google Patents. 1986.

3. Claflin ES, Bareket N. Configuring an electrostatic membrane mirror by least-squares fitting with analytically derived influence functions. J Opt Soc Am A. 1986;3:1833. https://doi.org/10.1364/ JOSAA.3.001833. 
4. Bille J, Jahn G, Frieben M. Modal control for wavefront reconstruction in adaptive optics. In: Barr LD, Burbidge $\mathrm{G}$, editors. International conference on advanced technology optical telescopes. Washington: International Society for Optics and Photonics; 1982. p. 269-75.

5. Dreher AW, Bille JF, Weinreb RN. Active optical depth resolution improvement of the laser tomographic scanner. Appl Opt. 1989;28:804. https://doi. org/10.1364/AO.28.000804.

6. Liang J, Grimm B, Goelz S, Bille JF. Objective measurement of wave aberrations of the human eye with the use of a Hartmann-Shack wave-front sensor. J Opt Soc Am A. 1994;11:1949. https://doi.org/10.1364/ JOSAA.11.001949.

7. Liang J, Williams DR, Miller DT. Supernormal vision and high-resolution retinal imaging through adaptive optics. J Opt Soc Am A Opt Image Sci Vis. 1997;14:2884-92.

8. Mrochen M, Kaemmerer M, Seiler T. Wavefrontguided laser in situ keratomileusis: early results in three eyes. J Refract Surg. 2000;16:116-21.

9. Bille JF, Agopov M, Alvarez-Diez C, et al. Compact adaptive optics system for multiphoton fundus imaging. J Mod Opt. 2008;55:749-58. https://doi. org/10.1080/09500340701608024.

10. Hermann B, Fernández EJ, Unterhuber A, et al. Adaptive-optics ultrahigh-resolution optical coherence tomography. Opt Lett. 2004;29:2142. https://doi. org/10.1364/OL.29.002142.

11. Roorda A, Romero-Borja F, Donnelly WJ III, et al. Adaptive optics scanning laser ophthalmoscopy. Opt Express. 2002;10:405. https://doi.org/10.1364/ OE.10.000405.

12. Resan M, Vukosavljevi M, Milivojevi M. Wavefront aberrations. In: Advances in ophthalmology. Croatia: InTech; 2012.

13. Bille JF, Grimm B, Liang J, Mueller K. Imaging of the retina by scanning laser tomography. New methods in microscopy and low light imaging. SPIE. 1989;1161:417.
14. Liang J. A new method to precisely measure the Wave Aberrations of the Human Eye with a HartmannShack Sensor. PhD thesis, University of Heidelberg, Germany. 1991.

15. Kasper M, Looze DP, Hippler S, et al. ALFA: adaptive optics for the Calar Alto observatory optics, control systems, and performance. Exp Astron. 2000;10:4973. https://doi.org/10.1023/A:1008165402336.

16. Droste D, Bille J. An ASIC for Hartmann-Shack wavefront detection. IEEE J Solid State Circuits. 2002;37:173-82. https://doi.org/10.1109/4.982423.

17. Bille JF. Method for programming an active mirror to mimic a wavefront. US Patent 6,220,707 B1. 2001.

18. von Pape U. Wavefront sensing in the human eye. PhD thesis, University of Heidelberg, Germany. 2002.

19. Campbell FW, Green DG. Optical and retinal factors affecting visual resolution. J Physiol. 1965;181:576-93.

20. Parthasarathy MK, Lakshminarayanan V. A brief history of aberrometry applications in ophthalmology and vision science. Singapore: Springer; 2017. p. 31-9.

21. Porter J. Adaptive optics for vision science: principles, practices, design, and applications. New York: Wiley-Interscience; 2006.

22. Burns SA, Marcos S, Elsner AE, Bara S. Contrast improvement of confocal retinal imaging by use of phase-correcting plates. Opt Lett. 2002;27:400-2.

23. Zhang Y, Rha J, Jonnal RS, Miller DT. Adaptive optics parallel spectral domain optical coherence tomography for imaging the living retina. Opt Express. 2005;13:4792. https://doi.org/10.1364/ OPEX.13.004792.

24. Roorda A, Williams DR. The arrangement of the three cone classes in the living human eye. Nature. 1999;397:520-2. https://doi.org/10.1038/17383.

25. Bille JF. The development of wavefront technology and its application to ophthalmology. In: Aberrationfree refractive surgery. Berlin, Heidelberg: Springer Berlin Heidelberg; 2003. p. 1-23.

Open Access This chapter is licensed under the terms of the Creative Commons Attribution 4.0 International License (http://creativecommons.org/licenses/by/4.0/), which permits use, sharing, adaptation, distribution and reproduction in any medium or format, as long as you give appropriate credit to the original author(s) and the source, provide a link to the Creative Commons license and indicate if changes were made.

The images or other third party material in this chapter are included in the chapter's Creative Commons license, unless indicated otherwise in a credit line to the material. If material is not included in the chapter's Creative Commons license and your intended use is not permitted by statutory regulation or exceeds the permitted use, you will need to obtain permission directly from the copyright holder.

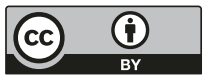

